\title{
On analyticity rate estimates to the magneto-hydrodynamic equations in Besov-Morrey spaces
}

Minghua Yang*

"Correspondence:

ymh20062007@163.com School of Mathematics and

Computational Science, Sun Yat-sen University, No. 135, Xingang Xi Road, Guangzhou, 510275, P.R. China

\section{Springer}

\begin{abstract}
In this article, we establish higher-order regularizing rate estimates of solutions to generalized magneto-hydrodynamic equations in Morrey spaces with initial data $\left(u_{0}, d_{0}\right)$ in Besov-Morrey spaces $\dot{\mathbf{N}}_{r, \lambda, \infty}^{-s} \times \dot{\mathbf{N}}_{r, \lambda, \infty}^{-s}$, where $n \geq 2,1 \leq r<\infty, 0 \leq \lambda<n$, $r>n-\lambda, \frac{1}{2}+\frac{n-\lambda}{4 r}<\sigma<1+\frac{n-\lambda}{4 r}$, and $s=2 \sigma-1-\frac{n-\lambda}{r}$, for which under some smallness condition, the solution of the Cauchy problem is analytic in the spatial variable. Our class of initial data contains strongly singular functions and measures and extends the ones in early work.
\end{abstract}

MSC: 76B03; 76D03

Keywords: magneto-hydrodynamic equations; Besov-Morrey spaces; regularizing rate estimates; Morrey spaces

\section{Introduction and main results}

In this article, we investigate the generalized magneto-hydrodynamic equations in the whole space $\mathbb{R}^{n}$,

$$
\left\{\begin{array}{l}
u_{t}+u \cdot \nabla u+(-\Delta)^{\sigma} u-d \cdot \nabla d+\nabla p=0 \quad \text { in } \mathbb{R}^{n} \times(0,+\infty) \\
\nabla \cdot u=0, \quad \nabla \cdot d=0, \\
d_{t}+u \cdot \nabla d+(-\Delta)^{\sigma} d-d \cdot \nabla u=0 \quad \text { in } \mathbb{R}^{n} \times(0,+\infty) \\
\left.(u, d)\right|_{t=0}=\left(u_{0}, d_{0}\right) .
\end{array}\right.
$$

Here $u$ is the velocity field of the flow, $d(\cdot, t)$ is the magnetic field. $p(\cdot, t): \mathbb{R}^{n} \rightarrow \mathbb{R}$ represents the pressure function. $\nabla \cdot u=0$ and $\nabla \cdot d=0$ represent the incompressible conditions. $\left(u_{0}, d_{0}\right)$ is for given initial data with $\nabla \cdot u_{0}=0$ and $\nabla \cdot d_{0}=0$ in the distribution sense.

When $\sigma=1$, the equations of system (1.1) become the usual MHD equations, which govern the dynamics of the velocity and magnetic fields in electrically conducting fluids. The system plays a fundamental role in applied sciences such as astrophysics, geophysics, and plasma physics. The first equation of system (1.1) reflects the conservation of momentum, the third equation of system (1.1) is the magnetic induction equation and the second equation of system (1.1) specifies the conservation of mass.

For general $\sigma$, system (1.1) is a generalization of the usual incompressible MHD system. As observed in [1], a fractional power of Laplacian can, in principle, be used as a

(c) 2015 Yang. This article is distributed under the terms of the Creative Commons Attribution 4.0 International License (http://creativecommons.org/licenses/by/4.0/), which permits unrestricted use, distribution, and reproduction in any medium, provided you give appropriate credit to the original author(s) and the source, provide a link to the Creative Commons license, and indicate if changes were made. 
mild dissipation in MHD equations. Besides their physical applications, system (1.1) is also mathematically significant.

According to Duhamel's principle, the mild solution $(u, d)$ for system $(1.1)$ can be represented as

$$
\left\{\begin{array}{l}
u=e^{-t \mathfrak{L}} u_{0}-\int_{0}^{t} e^{-(t-s) \mathfrak{L}} \mathbb{P} \nabla \cdot(u \otimes u-d \otimes d)(\cdot, s) d s, \\
d=e^{-t \mathfrak{L}} d_{0}-\int_{0}^{t} e^{-(t-s) \mathfrak{L}} \mathbb{P} \nabla \cdot(u \otimes d-d \otimes u)(\cdot, s) d s .
\end{array}\right.
$$

Here $\mathbb{P}$ is the Leray projection operator, which can be expressed as an $n \times n$ matrix: $\mathbb{P}=\left\{\mathbb{P}_{j, k}\right\}_{1 \leq j, k \leq n}=\left\{\delta_{j, k}+\mathbb{R}_{j} \mathbb{R}_{k}\right\}_{1 \leq j, k \leq n}$ with $\delta_{j, k}$ being the Kronecker symbol, $\mathbb{R}_{j}=\partial_{j}(-\Delta)^{-\frac{1}{2}}$ being the Riesz transform. $\mathfrak{L}:=(-\Delta)^{\sigma}$ denotes the fractional Laplacian, which is defined as $\left[\widehat{(-\Delta)^{\sigma} f}\right](\xi)=|\xi|^{2 \sigma} \hat{f}(\xi)$.

To give a clearer introduction to our results in this article, we first note that system (1.1) enjoys scaling properties. Clearly, if $(u(x, t), d(x, t))$ is a solution to system (1.1), then $\left(u^{\lambda}(x, t), d^{\lambda}(x, t)\right)$ is also a solution of (1.1) corresponding to the initial data $\left(u_{0}^{\lambda}, d_{0}^{\lambda}\right)$, where

$$
\begin{aligned}
& u^{\lambda}(x, t):=\lambda^{2 \sigma-1} u\left(\lambda x, \lambda^{2 \sigma} t\right), \quad d^{\lambda}(x, t):=\lambda^{2 \sigma-1} d\left(\lambda x, \lambda^{2 \sigma} t\right), \\
& u_{0}^{\lambda}(x):=\lambda^{2 \sigma-1} u_{0}(\lambda x), \quad d_{0}^{\lambda}(x):=\lambda^{2 \sigma-1} d_{0}(\lambda x) .
\end{aligned}
$$

We say that the solution $(u, d)$ is self-similar for system $(1.1)$, if $\left(u^{\lambda}, d^{\lambda}\right)=(u, d)$ for each $\lambda>0$.

A function space $\mathbb{Y}$ is called a critical space for (1.1) if it satisfies invariance under the scaling $\|u(\cdot, t)\|_{\mathbb{Y}}=\left\|u^{\lambda}(\cdot, t)\right\|_{\mathbb{Y}}$ for all $u \in \mathbb{Y}$.

Before going further, we recall the functional spaces we are going to use. Let $\mathscr{S}$ be the Schwartz class of rapidly decreasing functions and $\mathscr{S}^{\prime}$ be the space of tempered distributions. Here $\mathcal{F}$ and $\mathcal{F}^{-1}$ denote the Fourier and inverse Fourier transforms of $L^{1}$ functions, respectively, defined by $\mathcal{F} f=\hat{f}(\xi)=(2 \pi)^{-\frac{n}{2}} \int_{\mathbb{R}^{n}} e^{-i x \cdot \xi} f(x) d x$ and $\mathcal{F}^{-1} f=\check{f}(x)=$ $(2 \pi)^{-\frac{n}{2}} \int_{\mathbb{R}^{n}} e^{i x \cdot \xi} f(\xi) d \xi$. More generally, the Fourier transform of any $f \in \mathscr{S}^{\prime}$ is given by $(\mathcal{F} f, g)=(f, \mathcal{F} g)$, for any $g \in \mathscr{S}$. First, we recall the definition of Morrey space introduced in [2]: for $1 \leq p<\infty$ and $0 \leq \lambda<n$, the Morrey space $M_{p, \lambda}=M_{p, \lambda}\left(\mathbb{R}^{n}\right)$ is defined as

$$
M_{p, \lambda}:=\left\{f \in L_{\mathrm{loc}}^{p}\left(\mathbb{R}^{n}\right),\|f\|_{p, \lambda}<\infty\right\},
$$

with the norm given by

$$
\|f\|_{p, \lambda}:=\sup _{x_{0} \in \mathbb{R}^{n}} \sup _{r>0} r^{-\frac{\lambda}{p}}\left\{\int_{B\left(x_{0}, r\right)}|f(y)|^{p} d y\right\}^{\frac{1}{p}},
$$

where $B\left(x_{0}, r\right)$ denotes the ball in $\mathbb{R}^{n}$ with center $x_{0}$ and radius $r$. The space $M_{p, \lambda}$ endowed with the norm $\|\cdot\|_{p, \lambda}$ is a Banach space and has the following nice scaling property: for $\mu>0$,

$$
\|f(\mu x)\|_{p, \lambda}=\mu^{-\frac{n-\lambda}{p}}\|f(x)\|_{p, \lambda}
$$

Set $S_{h}=\left\{\phi \in \mathscr{S}, \partial^{\alpha} \mathcal{F} f(0)=0\right\}$ for any multi-index $\alpha \in \mathbb{N}_{0}:=\mathbb{N}^{n} \cup\{0\}, \mathbb{N}$ is the set of all positive integers. The dual space of $S_{h}$ is given by $S_{h}^{\prime}=\mathscr{S} / \mathcal{P}$, where $\mathcal{P}$ is the space of polynomials. We now introduce a dyadic partition of $\mathbb{R}^{n}$. Let $\varphi \in \mathscr{S}$ be a radially symmetric 
function with support in $\left\{\xi \in \mathbb{R}^{n}: \frac{3}{4} \leq|\xi| \leq \frac{8}{3}\right\}$ and such that

$$
\sum_{k=-\infty}^{\infty} \varphi_{k}(\xi)= \begin{cases}1, & \text { if } \xi \in \mathbb{R}^{n} \backslash\{0\} \\ 0, & \text { if } \xi=0\end{cases}
$$

Furthermore, we define $\varphi_{k}=\varphi\left(2^{-k} \xi\right)$ for every $k \in \mathbb{Z}$.

For any $f \in S_{h}^{\prime}$, setting $\Delta_{k} f=\left(\varphi_{k} \hat{f}\right)^{\check{\nu},}, k=0, \pm 1, \pm 2, \ldots$, and $S_{j} f=\sum_{k \leq j-1} \Delta_{k} f$. We have the Littlewood-Paley decomposition,

$$
f=\sum_{k=-\infty}^{\infty} \Delta_{k} f
$$

In [3], Kozono and Yamazaki introduced the homogeneous Besov-Morrey space $\dot{\mathbf{N}}_{p, \lambda, q}^{s}$. Recall that the space $\dot{\mathbf{N}}_{p, \lambda, q}^{s}$ is defined by

$$
\dot{\mathbf{N}}_{p, \lambda, q}^{s}=\left\{f \in S_{h}^{\prime}\left(\mathbb{R}^{n}\right):\|f\|_{\dot{\mathbf{N}}_{p, \lambda, q}^{s}}<\infty\right\}
$$

where

$$
\|f\|_{\dot{\mathbf{N}}_{p, \lambda, q}^{s}}= \begin{cases}\left(\sum_{k \in \mathbb{Z}}\left(2^{k s}\left\|\Delta_{k} f\right\|_{p, \lambda}\right)^{q}\right)^{\frac{1}{q}}, & \text { if } 1 \leq p \leq \infty, 1 \leq q<\infty, s \in \mathbb{R} \\ \sup _{k \in \mathbb{Z}} 2^{k s}\left\|\Delta_{k} f\right\|_{p, \lambda}, & \text { if } 1 \leq p \leq \infty, q=\infty, s \in \mathbb{R}\end{cases}
$$

When $\lambda=0, \dot{\mathbf{N}}_{p, 0, q}^{s}=\dot{\mathbf{B}}_{p, q}^{s}$, where $\dot{\mathbf{B}}_{p, q}^{s}$ is the homogeneous Besov space (see [4]).

If $\sigma=1, d=0$, system (1.1) is the well-known Navier-Stokes equations (NS), Foias and Temam [5] proved spatial analyticity for solutions in Sobolev spaces of periodical functions in an elementary way. The analyticity of solutions in $L^{p}$ for NS was first shown by Grujič, and Kukavica [6] and Lemarié-Rieusset [7] gave a different approach based on multilinear singular integrals. In a very interesting paper [8], Kahane established the spatial analyticity of weak solutions in Serrin's class $L_{t}^{p} L_{x}^{q}$ with $n / q+2 / p<1$. In cylindrical domains, Komatsu [9] showed that the solutions have global spatial analyticity up to the boundary. Using iterative derivative estimates, in [10], Giga and Sawada considered the regularizing rates of the higher-order derivatives and analyticity for the NS for the initial velocity in $L^{n}$. Similar results for the Navier-Stokes equations have been established by Sawada [11] when initial value $u_{0} \in \dot{H}^{\frac{n}{2}-1}\left(\mathbb{R}^{n}\right)$ and by Miura and Sawada [12] when $u_{0} \in B M O^{-1}$. Recently, Bae et al. [13] obtained the analyticity of the solutions of NS for the sufficiently small initial data in critical Besov spaces $\dot{\mathbf{B}}_{p, q}^{-1+3 / p}$, and Huang and Wang [14] showed the analyticity of the local solutions of NS with large initial data in critical Besov spaces $\dot{\mathbf{B}}_{p, q}^{-1+n / p}$ and modulation spaces $M_{p, 1}^{-1}$.

For general $\sigma$ and $d=0$, the equations of system (1.1) reduce to generalized NavierStokes equations (GNS). Dong and Li [15] showed that solutions are analytic in space variables for $1 / 2<\sigma<1$ with initial data in $L^{n /(2 \sigma-1)}$. Huang and Wang [14] showed the analyticity of the solutions of GNS in critical Besov spaces $\dot{\mathbf{B}}_{p, q}^{1-2 \sigma+n / p}$ and modulation spaces $M_{p, 1}^{1-2 \sigma}$ for $1 / 2<\sigma<1$. When $\sigma=\frac{1}{2}$, Huang and Wang [14] showed the analyticity of the solutions of GNS in critical Besov spaces $\dot{\mathbf{B}}_{p, 1}^{n / p}$ and modulation spaces $M_{\infty, 1}^{0} \cap \dot{\mathbf{B}}_{\infty, 1}^{0}$.

When $\sigma=1$, Liu and Cui in [16] show the analyticity of the usual MHD with initial data in $L^{n}, \dot{H}^{\frac{n}{2}-1}$ and $B M O^{-1}$. When $1 / 2<\sigma<(n+2) / 4$, Liu et al. in [17] show that the solution is analytic in the spatial variable of system (1.1) with the initial velocity in $P M^{n-2 \sigma+1}$. 
In [18], Yamamoto considered the regularizing rates and analyticity for the driftdiffusion equation for the initial data in $L^{\frac{n}{\theta}}(1<\theta \leq n)$ and extended the results to BesovMorrey spaces and Triebel-Lizorkin-Morrey spaces.

Inspired by the interesting work above, especially $[10-12,16-18]$ and motivated by the work of Mazzucato [19], and Kozono and Yamazaki [3] on the Navier-Stokes equations and a particular class of semi-linear heat equations with initial data in a certain Besov-Morrey space, our goal in the present article is to establish regularizing decay rate estimates and show space analyticity of mild solutions of system (1.1) with initial data in certain BesovMorrey spaces. For more information on Besov-Morrey spaces, we also refer to [20-22]. The question of the largest Besov-type spaces on initial data for which the solutions of (1.1) have well-posedness and analyticity is still open.

We give our main results in the following theorem.

Theorem 1.1 Let $n \geq 2,1 \leq r<\infty, 0 \leq \lambda<n, r>n-\lambda, \frac{1}{2}+\frac{n-\lambda}{4 r}<\sigma<1+\frac{n-\lambda}{4 r}, s=2 \sigma-1-\frac{n-\lambda}{r}$, $\alpha=\frac{2 \sigma-1}{\sigma}-\frac{n-\lambda}{2 r \sigma}, \nabla \cdot u_{0}=0, \nabla \cdot d_{0}=0,\left(u_{0}, d_{0}\right) \in \dot{\mathbf{N}}_{r, \lambda, \infty}^{-s} \times \dot{\mathbf{N}}_{r, \lambda, \infty}^{-s}, q \in[r, \infty]$. Assume further that there exist positive constants $M_{1}$ and $M_{2}$, such that the solutions $(u, d)$ to system (1.1) exist globally in time and satisfy

$$
\left\|\left(u_{0}, d_{0}\right)\right\|_{\dot{\mathbf{N}}_{r, \lambda, \infty}^{-s}} \leq M_{1}<+\infty, \quad \sup _{t>0} t^{\frac{\alpha}{2}}\|(u, d)\|_{2 r, \lambda} \leq M_{2}<+\infty
$$

for any $t>0$ and $M_{1}$ sufficiently small. Then there exist positive constants $K_{1}, K_{2}$ such that

$$
\left\|\left(\nabla^{m} u, \nabla^{m} d\right)\right\|_{q, \lambda} \leq K_{1}\left(K_{2}|\tilde{\beta}|\right)^{2 m} t^{-\frac{m}{2 \sigma}-\frac{1}{2 \sigma}\left(2 \sigma-1-\frac{n-\lambda}{q}\right)}
$$

where $\tilde{\beta} \in \mathbb{N}_{0}^{n}$ is a multi-index and $|\tilde{\beta}|=m$.

Remarks (I) The assumptions in Theorem 1.1 are natural. Indeed, let $1 \leq r<\infty, 0 \leq \lambda<n$, $r>n-\lambda, \frac{1}{2}+\frac{n-\lambda}{4 r}<\sigma<1+\frac{n-\lambda}{4 r}, \alpha=\frac{2 \sigma-1}{\sigma}-\frac{n-\lambda}{2 r \sigma}, s=2 \sigma-1-\frac{n-\lambda}{r}$. The Banach spaces $E$ are defined by $E=\left\{u: \nabla \cdot u=0, u \in B C\left((0, \infty), \dot{\mathbf{N}}_{r, \lambda, \infty}^{-s}\right), t^{\frac{\alpha}{2}} u \in B C\left((0, \infty), M_{2 r, \lambda}\right)\right\}$, which are Banach spaces with norms given by $\|u\|_{E}=\sup _{t>0}\|u(t)\|_{\dot{N}_{r, \lambda, \infty}^{-s}}+\sup _{t>0} t^{\frac{\alpha}{2}}\|u(t)\|_{2 r, \lambda}$. Let $u_{0}$ and $d_{0}$ be divergence free vector fields and $\left(u_{0}, d_{0}\right) \in \dot{\mathbf{N}}_{r, \lambda, \infty}^{-s} \times \dot{\mathbf{N}}_{r, \lambda, \infty}^{-s}$ with $\left\|\left(u_{0}, d_{0}\right)\right\|_{\dot{\mathbf{N}}_{r, \lambda, \infty}^{-s}}$ sufficiently small. Following a similar method to Theorems 3 and 4 on p.967 in [3] for Navier-Stokes equations, then there exists a globally in time solution $(u(x), d(x)) \in E \times E$ to (1.1) that satisfies (1.5). The proof of this is standard by making minor modifications with Theorems 3 and 4 on p.967 in [3], and we will outline its proof in the Appendix for completeness.

(II) When $\frac{1}{2}<\delta \leq 1, K_{2} \geq 2$, the estimate (1.6) is equivalent to (see [10])

$$
\left\|\left(\nabla^{m} u, \nabla^{m} d\right)\right\|_{q, \lambda} \leq K_{1}\left(K_{2}|\tilde{\beta}|\right)^{2 m-\delta} t^{-\frac{m}{2 \sigma}-\frac{1}{2 \sigma}\left(2 \sigma-1-\frac{n-\lambda}{q}\right)}
$$

(III) From Remark 1.4 of [23], we get $P M^{n-2 \sigma+1} \subset \dot{\mathbf{B}}_{p, \infty}^{1-2 \sigma+\frac{n}{p}}$ for $\frac{n}{2 \sigma-1}<p<\infty$. It follows (see [3], p.964) that the space $\dot{\mathbf{N}}_{r, \lambda, \infty}^{-2 \sigma+1+\frac{n-\lambda}{r}}$ is strictly larger than $\dot{\mathbf{B}}_{p, \infty}^{1-2 \sigma+\frac{n}{p}}$, when $p=\frac{n r}{n-\lambda}, \lambda>0$. The pseudomeasure space $P M^{a}(a \geq 0)$ introduced in [24] is defined as $P M^{a}:=\left\{f \in \mathscr{S}^{\prime}\right.$ :

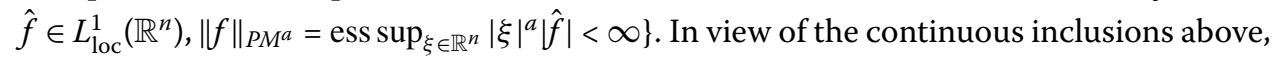
we see that the initial spaces $\dot{\mathbf{N}}_{r, \lambda, \infty}^{-s}\left(r>\max \left\{\frac{n-\lambda}{2 \sigma-1}, n-\lambda\right\}, \lambda>0\right)$ defined in Theorem 1.1 
is larger than pseudomeasure space $P M^{n-2 \sigma+1}$ in [17]. In [17], the authors considered the regularizing rates of the higher-order derivatives for system (1.1) for the initial velocity in $P M^{n-2 \sigma+1}$.

(IV) In particular, when $\sigma=1, d(x, t)=0$, system (1.1) becomes the usual Navier-Stokes equations. We also notice that $B M O^{-1}$ may be regarded as the largest critical space for initial data, where well-posedness and spatial analyticity of the Navier-Stokes equations can be constructed (see [25]). In [12], Miura and Sawada considered the regularizing rates of the higher-order derivatives for the Navier-Stokes equations for the initial velocity $u_{0} \in$ $B M O^{-1}$. The space $B M O^{-1}$ is the space of tempered distributions that can be written as divergence of a vector with components in $B M O$, where $B M O$ is the space of functions of bounded mean oscillations. The norm on $B M O^{-1}$ is given by

$$
\|f\|_{B M O^{-1}}:=\sup _{x_{0} \in \mathbb{R}^{n}} \sup _{r>0}\left(r^{-n} \int_{B\left(x_{0}, r\right)} \int_{0}^{r^{2}}\left|e^{t \Delta} f(y)\right|^{2}\right)^{\frac{1}{2}} .
$$

But the initial data $\dot{\mathbf{N}}_{r, \lambda, \infty}^{-s}(\sigma=1)$ given in Theorem 1.1 is not included completely with the space $B M O^{-1}$. Using the characterization from Lemma 2.3 below, we obtain (see [19], p.1314)

$$
\dot{\mathbf{N}}_{r, \lambda, \infty}^{\frac{n-\lambda}{r}-1} \subset B M O^{-1}, \quad r \geq 2, n \geq 2,0 \leq \lambda<n, r>n-\lambda,
$$

and, for $0 \leq \lambda<n, n \geq 2$,

$$
\dot{\mathbf{N}}_{1, \lambda, \infty}^{n-1-\lambda} \nsubseteq B M O^{-1}, \quad B M O^{-1} \nsubseteq \dot{\mathbf{N}}_{1, \lambda, \infty}^{n-1-\lambda} .
$$

Thus we note that even for the Navier-Stokes equations, our result in Theorem 1.1 is also new.

Notation Throughout this article, we denote vector fields $u=\left(u_{1}, u_{2}, \ldots, u_{n}\right), d=\left(d_{1}, d_{2}\right.$, $\left.\ldots, d_{n}\right)$. For a functional space $X$, we denote by $\|(u, d)\|_{X}$,

$$
\|d\|_{X}:=\sum_{i}^{n}\left\|d_{i}\right\|_{X}, \quad\|u\|_{X}:=\sum_{i}^{n}\left\|u_{i}\right\|_{X}, \quad\|(u, d)\|_{X}:=\|u\|_{X}+\|d\|_{X} .
$$

We use $c>0$ to denote a constant independent of the main variables, which may be different from line to line. We will employ the notation $a \lesssim b$ to mean that $a \leq c b$ for a universal constant $c>0$ that only depends on the parameters coming from the problems.

\section{Preliminaries}

In this section, we prepare several tools from harmonic analysis to be used in the proof of Theorem 1.1.

Lemma 2.1 Assume that $1 \leq p_{j}, q \leq \infty$ for all $j=1,2,3$, and $1 \leq p \leq \infty, 1 \leq r<\infty, 0 \leq \lambda$, $\lambda_{i}<$ for all $i=1,2,3$.

(1) If $p_{1}>p_{2}, s_{1}-\frac{n-\lambda}{p_{1}}=s_{2}-\frac{n-\lambda}{p_{2}}, s_{1}, s_{2} \in \mathbb{R}$, then

$$
\dot{\mathbf{N}}_{p_{2}, \lambda, q}^{s_{2}} \hookrightarrow \dot{\mathbf{N}}_{p_{1}, \lambda, q}^{s_{1}} \quad \text { and } \quad \dot{\mathbf{N}}_{r, \lambda, 1}^{0} \hookrightarrow M_{r, \lambda} \hookrightarrow \dot{\mathbf{N}}_{r, \lambda, \infty}^{0} .
$$

(2) If $1 \leq r \leq \tilde{r} \leq \infty, s \in \mathbb{R}$, then $\dot{\mathbf{N}}_{p, \lambda, r}^{s} \hookrightarrow \dot{\mathbf{N}}_{p, \lambda, \tilde{r}}^{s}$. 
(3) If $1 \leq p_{1} \leq p_{2} \leq \infty, 0 \leq \lambda_{1}, \lambda_{2}<n, \frac{n-\lambda_{1}}{p_{1}}=\frac{n-\lambda_{2}}{p_{2}}$, then $M_{p_{2}, \lambda_{2}} \subset M_{p_{1}, \lambda_{1}}$.

(4) If $\frac{1}{p_{3}}=\frac{1}{p_{2}}+\frac{1}{p_{1}}, \frac{\lambda_{3}}{p_{3}}=\frac{\lambda_{2}}{p_{2}}+\frac{\lambda_{1}}{p_{1}}, h_{i} \in M_{p_{i}, \lambda_{i}}$ for $i=1,2$, then

$\left\|h_{1} h_{2}\right\|_{p_{3}, \lambda_{3}} \leq\left\|h_{1}\right\|_{p_{1}, \lambda_{1}}\left\|h_{2}\right\|_{p_{2}, \lambda_{2}}$.

Proof For the proof of Lemma 2.1, we refer to [2, 3, 19, 26, 27].

From the Calderón-Zygmund operator theory, the Riesz transform $\mathbb{R}_{j}$ is continuous on $M_{r, v}$ for $1<r<\infty$ and $0 \leq v<n$, thus $\mathbb{P}$ is bounded on $M_{r, v}$. By the estimates for the multiplier operator, we can also see that $\mathbb{P}$ is bounded on $\dot{\mathbf{N}}_{p, \lambda, q}^{s}$ for $1 \leq p, q \leq \infty, 0 \leq \lambda<n$, and $s \in \mathbb{R}$.

Lemma 2.2 Let $\mu>0$ and $\mathbb{N}_{0}^{n} \ni \alpha=\left(\alpha_{1}, \alpha_{1}, \ldots, \alpha_{n}\right)$ be a multi-index with $|\alpha|=\mu, s_{1} \leq s_{2}$, $1 \leq q \leq \infty, 1 \leq p_{1} \leq p_{2} \leq \infty, 0 \leq \lambda<n$, for all $f \in \mathscr{S}^{\prime}$, then there exist $c_{0}, c_{1}, \tilde{c}, \tilde{c}_{0}, \tilde{c}_{1}, c$, and $\bar{c}$ depending only on $n$ such that

$$
\begin{aligned}
& \left\|e^{-t \mathfrak{L}} f\right\|_{p_{2}, \lambda} \leq \bar{c} t^{-\frac{1}{2 \sigma}\left(\frac{n-\lambda}{p_{1}}-\frac{n-\lambda}{p_{2}}\right)}\|f\|_{p_{1}, \lambda}, \\
& \left\|\partial^{\alpha} e^{-t \mathfrak{L}} f\right\|_{p_{2}, \lambda} \leq c_{0}\left(c_{1} \mu\right)^{\frac{\mu}{2 \sigma}} t^{-\frac{\mu}{2 \sigma}-\frac{1}{2 \sigma}\left(\frac{n-\lambda}{p_{1}}-\frac{n-\lambda}{p_{2}}\right)}\|f\|_{p_{1}, \lambda}, \\
& \left\|e^{-t \mathfrak{L}} f\right\|_{\dot{\mathbf{N}}_{p_{2}, \lambda, q}^{s_{2}}} \leq c t^{-\frac{s_{2}-s_{1}}{2 \sigma}-\frac{1}{2 \sigma}\left(\frac{n-\lambda}{p_{1}}-\frac{n-\lambda}{p_{2}}\right)}\|f\|_{\dot{N}_{p_{1}, \lambda, q}^{s_{1}},}, \\
& \left\|\partial^{\alpha} e^{-t \mathfrak{L} \mathfrak{L}}\right\|_{\dot{\mathbf{N}}_{p_{2}, \lambda, q}^{s_{2}}} \leq \tilde{c}_{0}\left(\tilde{c}_{1} \mu\right)^{\frac{\mu}{2 \sigma} \sigma} t^{-\frac{\mu+s_{2}-s_{1}}{2 \sigma}-\frac{1}{2 \sigma}\left(\frac{n-\lambda}{p_{1}}-\frac{n-\lambda}{p_{2}}\right)}\|f\|_{\dot{\mathbf{N}}_{p_{1}, \lambda, q}^{s_{1}}} .
\end{aligned}
$$

Further, if $s<\rho$, the estimate

$$
\left\|e^{-t \mathfrak{L}} f\right\|_{\dot{\mathbf{N}}_{r, \lambda, 1}^{\rho}} \leq \tilde{c} t^{\frac{s-\rho}{2 \sigma}}\|f\|_{\dot{\mathbf{N}}_{r, \lambda, \infty}^{s}}
$$

holds for every $t>0$.

Lemma 2.2 still holds true with $(-\Delta)^{\mu}$ in place of $\partial^{\alpha}$.

Proof We first prove (2.1) by proceeding in the following way. For all $1 \leq p \leq \infty, 0 \leq \lambda<n$, $g \in M_{p, \lambda}, \phi \in L^{1}$, in Morrey spaces we have

$$
\|g * \phi\|_{p, \lambda} \leq\|\phi\|_{L^{1}}\|g\|_{p, \lambda} .
$$

Note that (2.6) implies

$$
\left\|e^{-t \mathfrak{L}} f\right\|_{p_{2}, \lambda}=\left\|K_{t} * f\right\|_{p_{2}, \lambda} \leq\left\|K_{t}\right\|_{L^{1}}\|f\|_{p_{2}, \lambda} .
$$

According to Lemma 2.1 of [28], we have $K_{t} \in L^{p}$ for $1 \leq p \leq \infty$, where $K(x):=$ $\left(\frac{1}{2 \pi}\right)^{\frac{n}{2}} \int_{\mathbb{R}^{n}} e^{i x \cdot \xi} e^{-|\xi|^{2 \sigma}} d \xi$ and $K_{t}:=t^{-\frac{n}{2 \sigma}} K\left(\frac{x}{t^{\frac{1}{2 \sigma}}}\right)$. Thus we get $\left\|e^{-t \mathfrak{L}} f\right\|_{p_{2}, \lambda} \leq \bar{c}\|f\|_{p_{2}, \lambda}$.

From Lemma 2.1 of [28], we have the point-wise estimate $|K(x)| \leq \bar{c}(1+|x|)^{-n-2 \sigma}$. Hölder's inequality yields $\left|e^{-t \mathfrak{L}} f\right|^{p_{1}} \leq \bar{c} K_{t} *|f|^{p_{1}}$. Therefore, one has

$$
\begin{aligned}
\left|e^{-t \mathfrak{L}} f(x)\right|^{p_{1}} & \leq \bar{c} \int_{\mathbb{R}^{n}}|f(y)|^{p_{1}}\left(1+|x-y| t^{-\frac{1}{2 \sigma}}\right)^{-n-2 \sigma} d y \\
& \leq \bar{c} t^{-\frac{n}{2 \sigma}} \int_{0}^{+\infty} \int_{\partial B(x, r)}|f(y)|^{p_{1}} d S_{y}\left(1+r t^{-\frac{1}{2 \sigma}}\right)^{-n-2 \sigma} r^{n-1} d r
\end{aligned}
$$




$$
\begin{aligned}
& \leq \bar{c} t^{-\frac{n}{2 \sigma}} \int_{0}^{+\infty}\left(1+r t^{-\frac{1}{2 \sigma}}\right)^{-n-2 \sigma} d \rho(r) \\
& \leq \bar{c} t^{-\frac{n}{2 \sigma}} \int_{0}^{+\infty}(-\rho(r)) d\left(1+r t^{-\frac{1}{2 \sigma}}\right)^{-n-2 \sigma} \\
& \leq \bar{c} t^{-\frac{n}{2 \sigma}} t^{-\frac{1}{2 \sigma}}\|f\|_{p_{1}, \lambda}^{p_{1}} \int_{0}^{+\infty} r^{\lambda}\left(1+r t^{-\frac{1}{2 \sigma}}\right)^{-n-2 \sigma-1} d r \\
& \leq \bar{c} t^{\frac{\lambda-n}{2 \sigma}}\|f\|_{p_{1}, \lambda}^{p_{1}} .
\end{aligned}
$$

Using the definition of Morrey spaces in (1.4), we get

$$
\rho(r):=\int_{B(x, r)}|f(y)|^{p_{1}} d y \leq\|f\|_{p, \lambda}^{p_{1}} r^{\lambda}
$$

Then from the interpolation inequality it follows that

$$
\begin{aligned}
\left\|e^{-t \mathfrak{L}} f\right\|_{L^{p_{2}(B(x, r))}} & \leq\left\|e^{-t \mathfrak{L}} f\right\|_{L^{\infty}}^{1-\frac{p_{1}}{p_{2}}}\left\|e^{-t \mathfrak{L}} f\right\|_{L^{p_{1}}}^{\frac{p_{1}}{p_{2}}} \\
& \leq\left\|e^{-t \mathfrak{L}} f\right\|_{L^{\infty}}^{1-\frac{p_{1}}{p_{2}}}\left\|e^{-t \mathfrak{L}} f\right\|_{p, \lambda}^{\frac{p_{1}}{p_{2}}} r^{\frac{\lambda}{p_{2}}}
\end{aligned}
$$

Combining (2.7) and (2.8) gives

$$
\begin{aligned}
\left\|e^{-t \mathfrak{L}} f\right\|_{p_{2}, \lambda} & \leq\left\|e^{-t \mathfrak{L}} f\right\|_{L^{\infty}}^{1-\frac{p_{1}}{p_{2}}}\left\|e^{-t \mathfrak{L}} f\right\|_{p_{1}, \lambda}^{\frac{p_{1}}{p_{2}}} \\
& \leq \bar{c} t^{\frac{\lambda-n}{2 \sigma}\left(\frac{1}{p_{1}}-\frac{1}{p_{2}}\right)}\|f\|_{p_{1}, \lambda} .
\end{aligned}
$$

Thus, we complete the estimate (2.1).

To estimate (2.2), application of the commutativity of the semigroup and derivatives gives the following estimate:

$$
\partial^{\alpha} e^{-t \mathfrak{L}} f=\prod_{j=1}^{n}\left(\partial_{j} e^{-\frac{t}{2 \mu} \mathfrak{L}}\right)^{\alpha_{j}} e^{-\frac{t}{2} \mathfrak{L}} f
$$

Then, by (2.6),

$$
\left\|\partial^{\alpha} e^{-t \mathfrak{L}} f\right\|_{p_{2}, \lambda} \leq \prod_{j=1}^{n}\left\|\mathcal{F}^{-1}\left(i \xi_{j} e^{-\frac{t}{2 \mu}(|\xi|)^{2 \sigma}}\right)\right\|_{L^{1}}^{\alpha_{j}}\left\|e^{-\frac{t}{2} \mathfrak{L}} f\right\|_{p_{2}, \lambda} .
$$

With the aid of the Hörmander-Mikhlin type estimate in [29], we obtain

$$
\left\|\mathcal{F}^{-1}\left(i \xi_{j} e^{-\frac{t}{2 \mu}(|\xi|)^{2 \sigma}}\right)\right\|_{L^{1}}=\left(c_{1} \mu\right)^{\frac{1}{2 \sigma}} t^{-\frac{1}{2 \sigma}}
$$

Applying (2.10) and (2.11), we get

$$
\left\|\partial^{\alpha} e^{-t \mathfrak{L}} f\right\|_{p_{2}, \lambda} \leq c_{0}\left(c_{1} \mu\right)^{\frac{\mu}{2 \sigma}} t^{-\frac{\mu}{2 \sigma}-\frac{1}{2 \sigma}\left(\frac{n-\lambda}{p_{1}}-\frac{n-\lambda}{p_{2}}\right)}\|f\|_{p_{1}, \lambda} .
$$

Thus, one obtains the estimate of (2.2). 
To estimate (2.3), we apply the frequency projection operator $\Delta_{j}$ to $e^{-t \mathfrak{L}}$ and take the $M_{p, \lambda}$ norm, then by (2.1)

$$
\left\|\Delta_{j} e^{-t \mathfrak{L}} f\right\|_{p_{2}, \lambda} \leq c t^{-\frac{1}{2 \sigma}\left(\frac{n-\lambda}{p_{1}}-\frac{n-\lambda}{p_{2}}\right)}\left\|\Delta_{j} e^{-\frac{t}{2} \mathfrak{L}} f\right\|_{p_{1}, \lambda} .
$$

For every $j \in \mathbb{Z}$, it follows from (2.13) that

$$
\begin{aligned}
\left\|\Delta_{j} e^{-t \mathfrak{L}} f\right\|_{p_{2}, \lambda} & \leq c t^{-\frac{1}{2 \sigma}\left(\frac{n-\lambda}{p_{1}}-\frac{n-\lambda}{p_{2}}\right)}\left\|\Delta_{j} e^{-\frac{t}{2} \mathfrak{L}} f\right\|_{p_{1}, \lambda} \\
& \leq c t^{-\frac{1}{2 \sigma}\left(\frac{n-\lambda}{p_{1}}-\frac{n-\lambda}{p_{2}}\right)} t^{-\frac{s_{2}-s_{1}}{2 \sigma}} 2^{s_{1}-s_{2}}\left\|\Delta_{j} f\right\|_{p_{1}, \lambda}
\end{aligned}
$$

By the definition of Besov-Morrey spaces, from (2.14) we get (2.3) immediately.

For (2.4), using the estimate of (2.2), we can prove (2.4) exactly in the same way as deriving (2.3). Here we omit the proof of (2.4).

Assume that $s<\rho$, applying (2.3) with $q=\infty$, we obtain

$$
\left\|e^{-t \mathfrak{L}} f\right\|_{\dot{\mathbf{N}}_{r, \lambda, \infty}^{2 \rho-s}} \leq \tilde{c} t^{s-\rho} \frac{}{\sigma}\|\|_{\dot{\mathbf{N}}_{r, \lambda, \infty}^{s}}
$$

and

$$
\left\|e^{-t \mathfrak{L} f}\right\|_{\dot{\mathbf{N}}_{r, \lambda, \infty}^{s}} \leq \tilde{c}\|f\|_{\dot{\mathbf{N}}_{r, \lambda, \infty}^{s}}
$$

Using (2.15), (2.16), and the interpolation relation $\left(\dot{\mathbf{N}}_{r, \lambda, \infty}^{2 \rho-s}, \dot{\mathbf{N}}_{r, \lambda, \infty}^{s}\right)_{\frac{1}{2}, 1}=\dot{\mathbf{N}}_{r, \lambda, 1}^{\rho}$ (see Proposition 2.12 of [3]), we get the desired estimate (2.5). Thus, we complete the proof of Lemma 2.2.

Following the method used by [4], we give the proof of Lemma 2.3 .

Lemma 2.3 Suppose $1 \leq p, q \leq \infty, s>0$, and $0<\sigma<\infty$, then one has $f \in \dot{\mathbf{N}}_{p, \lambda, q}^{-2 s}$ if and only if

$$
\begin{cases}\left(\int_{0}^{\infty}\left(t^{\frac{s}{\sigma}}\left\|e^{-t \mathfrak{L}} f\right\|_{p, \lambda}\right)^{q} \frac{d t}{t}\right)^{\frac{1}{q}}, & \text { if } 1 \leq q<\infty \\ \sup _{t>0} t^{\frac{s}{\sigma}}\left(\left\|e^{-t \mathfrak{L}} f\right\|_{p, \lambda}\right), & \text { if } q=\infty\end{cases}
$$

Proof Let $\mathbb{C}=\left\{\xi: 0<r_{1} \leq|\xi| \leq r_{2}, r_{1}>0, r_{2}>0\right\}$ be an annulus, there exists a positive constant $c>0$, such that for any $1 \leq p \leq \infty$ and any couple $(t, \lambda)$ of positive real numbers, from the same ideas from Lemma 2.4 of [4], we have

$$
\operatorname{Supp} \hat{u} \subset \lambda \mathbb{C} \Rightarrow\left\|e^{-t \mathfrak{L}} u\right\|_{p, \lambda} \leq c e^{-c t \lambda^{2 \sigma}}\|u\|_{p, \lambda}
$$

here, we omit the proof (2.17).

In the following, we only show the case $1 \leq q<\infty$. For $q=\infty$ we have the same process. Note that, by (2.17),

$$
\left\|t^{\frac{s}{\sigma}} \Delta_{j} e^{-t \mathfrak{L}} f\right\|_{p, \lambda} \leq c t^{\frac{s}{\sigma}} e^{-c t 2^{2 j \sigma}}\left\|\Delta_{j} f\right\|_{p, \lambda} .
$$


Then, in virtue of $f=\sum_{j \in \mathbb{Z}} \Delta_{j} f$, we deduce that

$$
\left\|t^{\frac{s}{\sigma}} e^{-t \mathfrak{L}} f\right\|_{p, \lambda} \leq c\|f\|_{\dot{\mathrm{N}}_{p, \lambda, q}^{-2 s}} \sum t^{\frac{s}{\sigma}} 2^{2 j s} e^{-c t 2^{2 j \sigma}} c_{r, j}
$$
where $\left\{c_{r, j}=\frac{2^{-2 j s}\left\|\Delta_{j} f\right\|_{p, \lambda}}{\|f\|_{\dot{N}_{p, \lambda, q}^{-2 s}}^{-2,}}\right\}_{j \in \mathbb{Z}} \in l^{q}$. Note that $\left\|c_{r, j}\right\|_{l q}=1$, the change of variable $\tau=c t 2^{2 l \sigma}$
yields

$$
\begin{aligned}
\sum_{j \in \mathbb{Z}} t^{\frac{s}{\sigma}} 2^{2 j s} e^{-c t 2^{2 j \sigma}} & \leq \int_{-\infty}^{+\infty} t^{\frac{s}{\sigma}} 2^{2 l s} e^{-c t 2^{2 l \sigma}} d l \\
& =\int_{0}^{+\infty} t^{\frac{s}{\sigma}}\left(\frac{\tau}{c t}\right)^{\frac{s}{\sigma}} e^{-\tau} \frac{1}{c t 2^{2 \sigma l}(2 \sigma \log 2)} d \tau \\
& \left(\tau=c t 2^{2 l \sigma}, \frac{d \tau}{c t}=2^{2 l \sigma} \log \left(2^{2 \sigma}\right) d l\right) \\
& =\int_{0}^{+\infty} t^{\frac{s}{\sigma}}\left(\frac{\tau}{c t}\right)^{\frac{s}{\sigma}} e^{-\tau} \frac{1}{\frac{\tau}{2^{2 l \sigma}} 2^{2 \sigma l}(2 \sigma \log 2)} d \tau \quad\left(c t=\frac{\tau}{2^{2 l \sigma}}\right) \\
& =\frac{1}{2 \sigma c^{s / \sigma} \log 2} \int_{0}^{+\infty} t^{\frac{s}{\sigma}}\left(\frac{\tau}{t}\right)^{\frac{s}{\sigma}} e^{-\tau} \frac{1}{\tau} d \tau \\
& =\frac{1}{2 \sigma c^{s / \sigma} \log 2} \int_{0}^{+\infty} \tau^{\frac{s}{\sigma}-1} e^{-\tau} d \tau \\
& =\frac{1}{2 \sigma c^{s / \sigma} \log 2} \Gamma(s / \sigma),
\end{aligned}
$$

which is based on a technique developed in [30] (see (2.59) on p.27 in [30]), where $\Gamma(s)=$ $\int_{0}^{+\infty} x^{s-1} e^{-x} d x$ is the $\Gamma$ function for $s>0$.

Therefore, Hölder's inequality with weight $t^{\frac{s}{\sigma}} 2^{2 j s} e^{c t 2^{2 j \sigma}}$, Fubini's theorem, (2.19), and (2.20) imply that

$$
\begin{aligned}
& \int_{0}^{+\infty} t^{\frac{s}{\sigma}} e^{-t \mathfrak{L}}\|f\|_{p, \lambda}^{q} \frac{d t}{t} \\
& \quad \leq c\|f\|_{\dot{\mathrm{N}}_{p, \lambda, q}^{-2 s}} \int_{0}^{+\infty}\left(\sum_{j \in \mathbb{Z}} t^{\frac{s}{\sigma}} 2^{2 j s e^{-c t 2^{2 j \sigma}}}\right)^{q-1}\left(\sum_{j \in \mathbb{Z}} t^{\frac{s}{\sigma}} 2^{2 j s e^{-c t 2^{2 j \sigma}}} c_{r, j}^{q}\right) \frac{d t}{t} \\
& \quad \leq c\|f\|_{\dot{\mathbf{N}}_{p, \lambda}^{-2 s},} \sum_{j \in \mathbb{Z}} c_{r, j}^{q} \int_{0}^{+\infty} t^{\frac{s}{\sigma}} 2^{2 j s} e^{-c t 2^{2 j \sigma}} \frac{d t}{t} \\
& \quad \leq c\|f\|_{\dot{\mathbf{N}}_{p, \lambda, q}^{-2 s}} \int_{0}^{+\infty} t^{\frac{s}{\sigma}-1} e^{-t} d t \\
& \quad \lesssim\|f\|_{\dot{\mathbf{N}}_{p, \lambda, q}^{-2 s} .}
\end{aligned}
$$

Since $\Gamma\left(\frac{s}{\sigma}+1\right)=\int_{0}^{+\infty} t^{\frac{s}{\sigma}} e^{-t} d t$, by the definition of the Fourier transform, we thus get

$$
\Delta_{j} f=\Gamma\left(\frac{s}{\sigma}+1\right)^{-1} \int_{0}^{+\infty} t^{\frac{s}{\sigma}}(-\Delta)^{s+\sigma} e^{-t \mathfrak{L}} \Delta_{j} f d t
$$


Taking the $M_{p, \lambda}$ norm on (2.21), in view of (2.17), one easily sees that

$$
\left\|\Delta_{j} f\right\|_{p, \lambda} \lesssim \Gamma\left(\frac{s}{\sigma}+1\right)^{-1} \int_{0}^{+\infty} t^{\frac{s}{\sigma}} 2^{2 j s+2 j \sigma} e^{-\frac{c t}{2} 2^{2 j \sigma}}\left\|e^{-t \mathfrak{L}} f\right\|_{p, \lambda} d t
$$

The change of the variable $x=\frac{c t}{2} 2^{2 m \sigma}$ implies that

$$
\begin{aligned}
\sum_{j \in \mathbb{Z}} 2^{-2 j s q}\left\|\Delta_{j} f\right\|_{p, \lambda}^{q} & \lesssim \int_{0}^{+\infty} t^{\frac{s q}{\sigma}}\left\|e^{-t \mathfrak{L}} f\right\|_{p, \lambda}^{q}\left(\sum_{j \in \mathbb{Z}} 2^{2 j \sigma} e^{-\frac{c t}{2} 2^{2 j \sigma}} t\right) \frac{d t}{t} \\
& \lesssim \int_{0}^{+\infty} t^{\frac{s q}{\sigma}}\left\|e^{-t \mathfrak{L}} f\right\|_{p, \lambda}^{q}\left(\int_{0}^{+\infty} \frac{2 x}{c} e^{-x} \frac{1}{\sigma x \ln 2} d x\right) \frac{d t}{t} \\
& \lesssim \int_{0}^{+\infty} t^{\frac{s q}{\sigma}}\left\|e^{-t \mathfrak{L}} f\right\|_{p, \lambda}^{q} \frac{d t}{t}
\end{aligned}
$$

Thus, we complete the proof of Lemma 2.3.

Lemma 2.4 For all $\delta \in\left(\frac{1}{2}, 1\right)$, then there exists a constant $c>0$ such that

$$
\sum_{\tilde{\alpha} \leq \gamma}\left(\begin{array}{c}
\gamma \\
\tilde{\alpha}
\end{array}\right)|\tilde{\alpha}|^{|\tilde{\alpha}|-\delta}|\gamma-\tilde{\alpha}|^{|\gamma-\tilde{\alpha}|-\delta} \leq c|\gamma|^{|\gamma|-\delta}
$$

holds for every $\gamma \in \mathbb{Z}_{+}^{n},\left(\tilde{\alpha}_{1}, \tilde{\alpha}_{2}, \ldots, \tilde{\alpha}_{n}\right)=\tilde{\alpha} \leq \gamma=\left(\gamma_{1}, \gamma_{2}, \ldots, \gamma_{n}\right)$. Note that $\tilde{\alpha} \leq \gamma$ means $\tilde{\alpha}_{i} \leq \gamma_{i}$ for all $i=1,2, \ldots, n$ and $\left(\begin{array}{l}\gamma \\ \tilde{\alpha}\end{array}\right)=\prod_{j=1}^{n} \frac{\gamma_{j} !}{\tilde{\alpha}_{j} !\left(\gamma_{j}-\tilde{\alpha}_{j}\right) !}$.

Proof For the proof of Lemma 2.4, see [8].

Lemma 2.5 Let $\psi_{0}$ be a measurable and locally bounded function in $(0, T)$. Let $\left\{\psi_{j}\right\}_{j=1}^{\infty}$ be a sequence of measurable functions in $(0, T)$. Assume that $\alpha \in \mathbb{R}$ and $\mu, v>0$ satisfying $\mu+v=1$. Let $B_{\eta}>0$ be a number depending on $\eta \in(0,1)$, and assume that $B_{\eta}>0$ is nonincreasing with respect to $\eta$. Assume that there is a positive constant $\theta$ such that

$$
0 \leq \psi_{0}(t) \leq B_{\eta} t^{-\alpha}+\theta \int_{(1-\eta) t}^{t}(t-s)^{-\mu}(s)^{-v} \psi_{0}(s) d s
$$

and

$$
0 \leq \psi_{j+1}(t) \leq B_{\eta} t^{-\alpha}+\theta \int_{(1-\eta) t}^{t}(t-s)^{-\mu}(s)^{-v} \psi_{j}(s) d s
$$

for all $j \geq 0, t>0$ and $\eta \in(0,1)$. Let $\eta_{0}$ be a unique positive number such that $I\left(\eta_{0}\right)=$ $\min \left\{\frac{1}{2 \theta}, I(1)\right\}$ with $I(\eta)=\int_{(1-\eta) t}^{t}(t-s)^{-\mu}(s)^{-\nu-\alpha} d s$. Then

$$
\psi_{j}(t) \leq 2 B_{\tilde{\eta}} t^{-\alpha}
$$

for all $j \geq 0,0<\tilde{\eta} \leq \eta_{0}$, and $0<t<T$.

Proof For the proof of Lemma 2.5, we refer to [10]. 


\section{Proof of Theorem 1.1}

Before proving Theorem 1.1, we first follow the ideas from $[10,12]$ and prove a variant of Theorem 1.1 under some additional regularity assumptions.

Proposition 3.1 Suppose that the assumptions of Theorem 1.1 are satisfied. Assume furthermore that

$$
t^{\frac{m}{2 \sigma}+\frac{1}{2 \sigma}\left(2 \sigma-1-\frac{n-\lambda}{q}\right)}\left(\partial_{x}^{\tilde{\beta}} u, \partial_{x}^{\tilde{\beta}} d\right) \in L^{\infty}\left((0, \infty) ; M_{q, \lambda}\right)
$$

for all $r \leq q \leq \infty$. Then given $\frac{1}{2}<\delta \leq 1$, there exist constants $K_{1}>0, K_{2}>0$ (depending only on $n, M_{1}, M_{2}, \delta$, and $\left.\sigma\right)$, such that

$$
\left\|\left(\partial_{x}^{\tilde{\beta}} u, \partial_{x}^{\tilde{\beta}} d\right)\right\|_{q, \lambda} \leq K_{1}\left(K_{2} m\right)^{2 m-\delta} t^{-\frac{m}{2 \sigma}-\frac{1}{2 \sigma}\left(2 \sigma-1-\frac{n-\lambda}{q}\right)}
$$

for all $r \leq q \leq \infty$, where $|\tilde{\beta}|=m$.

Proof For $1 \leq p \leq \infty$ and $0 \leq \lambda<n$, by Lemma 2.2, note that (2.6), there exists a constant $c>0$ such that

$$
\left\|e^{-t \mathfrak{L}} \mathbb{P} \nabla f\right\|_{p, \lambda} \leq c t^{-\frac{1}{2 \sigma}}\|f\|_{p, \lambda}
$$

In fact, the proof of (3.3) is essentially the same as the proof of $\left\|e^{-t \Delta} \mathbb{P} \nabla f\right\|_{p} \leq c t^{-\frac{1}{2}}\|f\|_{p}$. The process of proving $\left\|e^{-t \Delta} \mathbb{P} \nabla f\right\|_{p} \leq c t^{-\frac{1}{2 \sigma}}\|f\|_{p}$ can be found in [2, 29].

Using Lemma 2.2 and (3.3), for $1 \leq p_{1} \leq p_{2} \leq \infty$ and $0 \leq \lambda<n$, a straightforward calculation yields the following elementary estimates:

$$
\left\|(-\Delta)^{\frac{\mu}{2}} e^{-t \mathfrak{L}} \mathbb{P} \nabla f\right\|_{p_{2}, \lambda} \leq c_{0}\left(c_{1} \mu\right)^{\frac{\mu}{2 \sigma}} t^{-\frac{\mu+1}{2 \sigma}-\frac{1}{2 \sigma}\left(\frac{n-\lambda}{p_{1}}-\frac{n-\lambda}{p_{2}}\right)}\|f\|_{p_{1}, \lambda}
$$

We use an induction argument with respect to $m$.

Step 1. We first shall prove (3.2) for $m=0$. Taking the $M_{q, \lambda}$ norm to the first term of (1.2), for some $\epsilon \in(0,1)$,

$$
\begin{aligned}
\|u(t)\|_{q, \lambda} \leq & \left\|e^{-t \mathfrak{L}} u_{0}\right\|_{q, \lambda}+\int_{0}^{(1-\epsilon) t}\left\|e^{-(t-\tau) \mathfrak{L}} \mathbb{P} \nabla(u \otimes u-d \otimes d)\right\|_{q, \lambda} d \tau \\
& +\int_{(1-\epsilon) t}^{t}\left\|e^{-(t-\tau) \mathfrak{L}} \mathbb{P} \nabla(u \otimes u-d \otimes d)\right\|_{q, \lambda} d \tau \\
:= & B_{1}+B_{2}+B_{3} .
\end{aligned}
$$

We shall estimate each term. To estimate the first term $B_{1}$ on the right side of (3.5), we note that, by (3.4),

$$
\begin{aligned}
B_{1} & \leq t^{-\frac{n-\lambda}{2 \sigma}\left(\frac{1}{r}-\frac{1}{q}\right)}\left\|e^{-\frac{t}{2} \mathfrak{L}} u_{0}\right\|_{r, \lambda} \\
& \leq t^{-\frac{n-\lambda}{2 \sigma}\left(\frac{1}{r}-\frac{1}{q}\right)}\left(\frac{t}{2}\right)^{-\frac{s}{2 \sigma}} \sup _{t>0}\left(\frac{t}{2}\right)^{\frac{s}{2 \sigma}}\left\|e^{-\frac{t}{2} \mathfrak{L}} u_{0}\right\|_{r, \lambda} \\
& \leq C t^{-\frac{1}{2 \sigma}\left(2 \sigma-1-\frac{n-\lambda}{q}\right)}\left\|u_{0}\right\|_{\dot{\mathrm{N}}_{r, \lambda, \infty}^{-s}} \\
& \leq C M_{1} t^{-\frac{1}{2 \sigma}\left(2 \sigma-1-\frac{n-\lambda}{q}\right)} .
\end{aligned}
$$


It follows from (4) of Lemma 2.1, (1.5), and (3.4) that

$$
\begin{aligned}
B_{2} & \leq \int_{0}^{(1-\epsilon) t}(t-\tau)^{-\frac{1}{2 \sigma}-\frac{n-\lambda}{2 \sigma}\left(\frac{1}{r}-\frac{1}{q}\right)}\left(\|u\|_{2 r, \lambda}^{2}+\|d\|_{2 r, \lambda}^{2}\right) d \tau \\
& \leq M_{2}^{2} \int_{0}^{(1-\epsilon) t}(t-\tau)^{-\frac{1}{2 \sigma}-\frac{n-\lambda}{2 \sigma}\left(\frac{1}{r}-\frac{1}{q}\right)} \tau^{-\alpha} d \tau \\
& \leq C M_{2}^{2} t^{-\frac{1}{2 \sigma}\left(2 \sigma-1-\frac{n-\lambda}{q}\right)} \epsilon^{-\frac{1}{2 \sigma}-\frac{n-\lambda}{2 \sigma}\left(\frac{1}{r}-\frac{1}{q}\right)}
\end{aligned}
$$

By Lemma 2.1, (1.5), and (3.4), similarly we can derive

$$
\begin{aligned}
B_{3} & \leq \int_{0}^{(1-\epsilon) t}(t-\tau)^{-\frac{1}{2 \sigma}-\frac{n-\lambda}{2 \sigma}\left(\frac{q+2 r}{2 r q}-\frac{1}{q}\right)}\|u \otimes u+d \otimes d\|_{\frac{2 r q}{q+2 r}, \lambda} d \tau \\
& \leq \int_{0}^{(1-\epsilon) t}(t-\tau)^{-\frac{1}{2 \sigma}-\frac{n-\lambda}{2 \sigma}\left(\frac{q+2 r}{2 r q}-\frac{1}{q}\right)}\left(\|u\|_{q, \lambda}\|u\|_{2 r, \lambda}+\|d\|_{q, \lambda}\|d\|_{2 r, \lambda}\right) d \tau \\
& \leq C M_{2} \int_{0}^{(1-\epsilon) t}(t-\tau)^{-\frac{1}{2 \sigma}-\frac{n-\lambda}{2 \sigma}\left(\frac{q+2 r}{2 r q}-\frac{1}{q}\right)} \tau^{-\frac{\alpha}{2}}\|(u, d)\|_{q, \lambda} d \tau
\end{aligned}
$$

Note that $\alpha=\frac{2 \sigma-1}{\sigma}-\frac{n-\lambda}{2 r \sigma}$, denoting $B_{\epsilon}=C M_{1}+C M_{2}^{2} \epsilon^{-\frac{1}{2 \sigma}-\frac{n-\lambda}{2 \sigma}\left(\frac{1}{r}-\frac{1}{q}\right)}$, and combining (3.6), (3.7), and (3.8), one obtains

$$
\begin{aligned}
\|u(t)\|_{q, \lambda} \leq & B_{\epsilon} t^{-\frac{1}{2 \sigma}\left(2 \sigma-1-\frac{n-\lambda}{q}\right)} \\
& +C M_{2} \int_{0}^{(1-\epsilon) t}(t-\tau)^{-\frac{1}{2 \sigma}-\frac{n-\lambda}{2 \sigma}\left(\frac{q+2 r}{2 r q}-\frac{1}{q}\right)} \tau^{-\frac{\alpha}{2}}\|(u, d)\|_{q, \lambda} d \tau .
\end{aligned}
$$

Similarly, we can get the desired estimate of $\|d\|_{q, \lambda}$,

$$
\begin{aligned}
\|d(t)\|_{q, \lambda} \leq & B_{\epsilon} t^{-\frac{1}{2 \sigma}\left(2 \sigma-1-\frac{n-\lambda}{q}\right)} \\
& +C M_{2} \int_{0}^{(1-\epsilon) t}(t-\tau)^{-\frac{1}{2 \sigma}-\frac{n-\lambda}{2 \sigma}\left(\frac{q+2 r}{2 r q}-\frac{1}{q}\right)} \tau^{-\frac{\alpha}{2}}\|(u, d)\|_{q, \lambda} d \tau .
\end{aligned}
$$

Thus, by (3.9) and (3.10),

$$
\begin{aligned}
\|(u(t), d(t))\|_{q, \lambda} \leq & B_{\epsilon} t^{-\frac{1}{2 \sigma}\left(2 \sigma-1-\frac{n-\lambda}{q}\right)} \\
& +C M_{2} \int_{0}^{(1-\epsilon) t}(t-\tau)^{-\frac{1}{2 \sigma}-\frac{n-\lambda}{2 \sigma}\left(\frac{q+2 r}{2 r q}-\frac{1}{q}\right)} \tau^{-\frac{\alpha}{2}}\|(u, d)\|_{q, \lambda} d \tau .
\end{aligned}
$$

Therefore, according to Lemma 2.5, we get (3.2) for $|\tilde{\beta}|=m=0$.

Step 2. We next consider the case $m=1$. The proof of (3.2) is essentially contained in Step 3. Thus here we omit the details.

Step 3. Assume that $m \geq 2$. We suppose that (3.2) holds for $q \in[r, \infty]$ and all $|\tilde{\beta}| \leq m-1$. We need to prove that (3.2) holds for $|\tilde{\beta}|=m$. Then, for $|\tilde{\beta}|=m$ and some $\epsilon \in(0,1)$, we see that

$$
\begin{aligned}
\left\|\partial^{\beta} u(t)\right\|_{q, \lambda} \leq & \left\|\partial^{\tilde{\beta}} e^{-t \mathfrak{L}} u_{0}\right\|_{q, \lambda} \\
& +\int_{0}^{(1-\epsilon) t}\left\|\partial^{\tilde{\beta}} e^{-(t-\tau) \mathfrak{L}} \mathbb{P} \nabla(u \otimes u-d \otimes d)\right\|_{q, \lambda} d \tau
\end{aligned}
$$




$$
\begin{aligned}
& +\int_{(1-\epsilon) t}^{t}\left\|\partial^{\tilde{\beta}} e^{-(t-\tau) \mathfrak{L}} \mathbb{P} \nabla(u \otimes u-d \otimes d)\right\|_{q, \lambda} d \tau \\
= & : A_{1}+A_{2}+A_{3} .
\end{aligned}
$$

We shall estimate each of the above terms $A_{1}, A_{2}, A_{3}$ separately. Note that $\frac{m}{2 \sigma} \leq 2 m-\delta$, since $m \geq 2$ and $0<\delta \leq 1$. Observe that by (1.5), and Lemmas 2.2 and 2.3

$$
\begin{aligned}
A_{1} & \leq t^{-\frac{n-\lambda}{2 \sigma}\left(\frac{1}{r}-\frac{1}{q}\right)}\left\|e^{-\frac{t}{2} \mathfrak{L}} u_{0}\right\|_{r, \lambda} \\
& \leq \tilde{C}_{1}\left(\tilde{C}_{2} m\right)^{\frac{m}{2 \sigma}} t^{-\frac{m}{2 \sigma}-\frac{n-\lambda}{2 \sigma}\left(\frac{1}{r}-\frac{1}{q}\right)}\left(\frac{t}{2}\right)^{-\frac{s}{2 \sigma}} \sup _{t>0}\left(\frac{t}{2}\right)^{\frac{s}{2 \sigma}}\left\|e^{-\frac{t}{2} \mathfrak{L}} u_{0}\right\|_{r, \lambda} \\
& \lesssim \tilde{C}_{1}\left(\tilde{C}_{2} m\right)^{\frac{m}{2 \sigma}} t^{-\frac{m}{2 \sigma}-\frac{1}{2 \sigma}\left(2 \sigma-1-\frac{n-\lambda}{q}\right)}\left\|u_{0}\right\|_{\dot{\mathbf{N}}_{r, \lambda, \infty}^{-\tilde{\lambda}}} \\
& \lesssim C_{1} M_{1}\left(C_{2} m\right)^{2 m-\delta} t^{-\frac{m}{2 \sigma}-\frac{1}{2 \sigma}\left(2 \sigma-1-\frac{n-\lambda}{q}\right)} .
\end{aligned}
$$

To estimate the term $A_{2}$, we note that, by Lemma 2.1, (1.5), and (3.4),

$$
\begin{aligned}
A_{2} & \leq \tilde{C}_{3}\left(\tilde{C}_{4} m\right)^{\frac{m}{2 \sigma}} \int_{0}^{(1-\epsilon) t}(t-\tau)^{-\frac{m+1}{2 \sigma}-\frac{n-\lambda}{2 \sigma}\left(\frac{1}{r}-\frac{1}{q}\right)}\|u \otimes u-d \otimes d\|_{r, \lambda} d \tau \\
& \leq \tilde{C}_{3}\left(\tilde{C}_{4} m\right)^{\frac{m}{2 \sigma}} M_{2}^{2} \int_{0}^{(1-\epsilon) t}(t-\tau)^{-\frac{m+1}{2 \sigma}-\frac{n-\lambda}{2 \sigma}\left(\frac{1}{r}-\frac{1}{q}\right)} \tau^{-\alpha} d \tau \\
& \leq C_{3}\left(C_{4} m\right)^{\frac{m}{2 \sigma}} M_{2}^{2} t^{-\frac{m}{2 \sigma}-\frac{1}{2 \sigma}\left(2 \sigma-1-\frac{n-\lambda}{q}\right)} \epsilon^{-\frac{m+1}{2 \sigma}-\frac{n-\lambda}{2 \sigma}\left(\frac{1}{r}-\frac{1}{q}\right)} \\
& \leq C_{3}\left(C_{4} m\right)^{2 m-\delta} M_{2}^{2} t^{-\frac{m}{2 \sigma}-\frac{1}{2 \sigma}\left(2 \sigma-1-\frac{n-\lambda}{q}\right)} \epsilon^{-\frac{m+1}{2 \sigma}-\frac{n-\lambda}{2 \sigma}\left(\frac{1}{r}-\frac{1}{q}\right)} .
\end{aligned}
$$

We now calculate $\nabla^{m}(u \otimes u-d \otimes d)$ by Leibniz's rule. Lemma 2.1 and (3.4) yield

$$
\begin{aligned}
A_{3} \leq & 2 C \int_{(1-\epsilon) t}^{t}(t-\tau)^{-\frac{1}{2 \sigma}}\left(\left\|\nabla^{m} u\right\|_{q, \lambda}\|u\|_{L^{\infty}}+\left\|\nabla^{m} d\right\|_{q, \lambda}\|d\|_{L^{\infty}}\right) d \tau \\
& +C \int_{(1-\epsilon) t}^{t}(t-\tau)^{-\frac{1}{2 \sigma}} \\
& \quad \times \max _{|\tilde{\beta}|=m} \sum_{0<\gamma<\tilde{\beta}}\left(\begin{array}{c}
\tilde{\beta} \\
\gamma
\end{array}\right)\left(\left\|\partial_{x}^{\gamma} u\right\|_{q, \lambda}\left\|\partial_{x}^{\tilde{\beta}-\gamma} u\right\|_{L^{\infty}}+\left\|\partial_{x}^{\gamma} d\right\|_{q, \lambda}\left\|\partial_{x}^{\tilde{\beta}-\gamma} d\right\|_{L^{\infty}}\right) d \tau \\
=: & A_{31}+A_{32} .
\end{aligned}
$$

Here, $\gamma<\tilde{\beta}$ means $\gamma_{i} \leq \tilde{\beta}_{i}$ and $|\gamma|<|\tilde{\beta}|$ for the multi-indices $\tilde{\beta}=\left(\tilde{\beta}_{1}, \tilde{\beta}_{2}, \ldots, \tilde{\beta}_{n}\right)$ and $\gamma=$ $\left(\gamma_{1}, \gamma_{2}, \ldots, \gamma_{n}\right)$, where $i=1,2, \ldots, n$.

In order to estimate the first term on the right hand of (3.13), according to Step 1, we note that there exists $c_{5}>0$ such that $\|(u, d)\|_{\infty, \lambda}=\|(u, d)\|_{L^{\infty}} \leq C_{5} K_{1} t^{\frac{1-2 \sigma}{2 \sigma}}$, then

$$
\begin{aligned}
A_{31} & \leq C_{5} K_{1} \int_{(1-\epsilon) t}^{t}(t-\tau)^{-\frac{1}{2 \sigma}} \tau^{\frac{1-2 \sigma}{2 \sigma}}\left(\left\|\nabla^{m} u\right\|_{q, \lambda}+\left\|\nabla^{m} d\right\|_{q, \lambda}\right) d \tau \\
& \leq C_{5} K_{1} \int_{(1-\epsilon) t}^{t}(t-\tau)^{-\frac{1}{2 \sigma}} \tau^{\frac{1-2 \sigma}{2 \sigma}}\left\|\left(\nabla^{m} u, \nabla^{m} d\right)\right\|_{q, \lambda} d \tau .
\end{aligned}
$$


By the assumption of the induction, we obtain

$$
\begin{aligned}
A_{32} \leq & C_{6} \int_{(1-\epsilon) t}^{t}(t-\tau)^{-\frac{1}{2 \sigma}} \max _{|\tilde{\beta}|=m} \sum_{0<\gamma<\tilde{\beta}}\left(\begin{array}{l}
\tilde{\beta} \\
\gamma
\end{array}\right) K_{1}\left(K_{2}|\tilde{\beta}-\gamma|\right)^{2|\tilde{\beta}-\gamma|-\delta} \tau^{-\frac{|\tilde{\beta}-\gamma|}{2 \sigma}-\frac{1}{2 \sigma}\left(2 \sigma-1-\frac{n-\lambda}{q}\right)} \\
& \times K_{1}\left(K_{2}|\gamma|\right)^{2|\gamma|-\delta} \tau^{-\frac{|\gamma|}{2 \sigma}-\frac{1}{2 \sigma}(2 \sigma-1)} d \tau \\
\leq & C_{6} K_{1}^{2} K_{2}^{2 m-2 \delta} \sum_{0<\gamma<\tilde{\beta}}\left(\begin{array}{l}
\tilde{\beta} \\
\gamma
\end{array}\right)|\tilde{\beta}-\gamma|^{2|| \tilde{\beta}-\gamma \mid-\delta}|\gamma|^{2|\gamma|-\delta} \\
& \times \int_{(1-\epsilon) t}^{t}(t-\tau)^{-\frac{1}{2 \sigma}} \tau^{-\frac{|\tilde{\beta}-\gamma|}{2 \sigma}-\frac{1}{2 \sigma}\left(2 \sigma-1-\frac{n-\lambda}{q}\right)} \tau^{-\frac{|\gamma|}{2 \sigma}-\frac{1}{2 \sigma}(2 \sigma-1)} d \tau \\
\leq & C_{6} K_{1}^{2} K_{2}^{2 m-2 \delta} \sum_{0<\gamma<\tilde{\beta}}\left(\begin{array}{l}
\tilde{\beta} \\
\gamma
\end{array}\right)|\tilde{\beta}-\gamma|^{2|| \tilde{\beta}-\gamma \mid-\delta}|\gamma|^{2|\gamma|-\delta} t^{-\frac{m}{2 \sigma}-\frac{1}{2 \sigma}\left(2 \sigma-1-\frac{n-\lambda}{q}\right)} \\
& \times \int_{(1-\epsilon) t}^{1}(1-\tau)^{-\frac{1}{2 \sigma}} \tau^{-\frac{m}{2 \sigma}-\frac{1}{2 \sigma}\left(4 \sigma-2-\frac{n-\lambda}{q}\right)} d \tau .
\end{aligned}
$$

Applying Lemma 2.4, it follows that

$$
A_{32} \leq C_{6} K_{1}^{2} K_{2}^{2 m-2 \delta} m^{2 m-\delta} t^{-\frac{m}{2 \sigma}-\frac{1}{2 \sigma}\left(2 \sigma-1-\frac{n-\lambda}{q}\right)} I(\epsilon),
$$

where $I(\epsilon):=\int_{1-\epsilon}^{1}(1-\tau)^{-\frac{1}{2 \sigma}} \tau^{-\frac{m}{2 \sigma}-\frac{1}{2 \sigma}\left(4 \sigma-2-\frac{n-\lambda}{q}\right)} d \tau$.

Note that we set

$$
\begin{aligned}
b_{\epsilon}= & C_{1} M_{1}\left(C_{2} m\right)^{2 m-\delta}+C_{3}\left(C_{4} m\right)^{2 m-\delta} M_{2}^{2} \epsilon^{-\frac{m+1}{2 \sigma}-\frac{n-\lambda}{2 \sigma}\left(\frac{1}{r}-\frac{1}{q}\right)} \\
& +C_{6} K_{1}^{2} K_{2}^{2 m-2 \delta} m^{2 m-\delta} I(\epsilon) .
\end{aligned}
$$

Combining the above estimates for (3.11), (3.12), (3.13), (3.14), (3.15), and (3.16), we obtain

$$
\left\|\nabla^{m} u(t)\right\|_{q, \lambda} \leq b_{\epsilon} t^{-\frac{m}{2 \sigma}-\frac{1}{2 \sigma}\left(2 \sigma-1-\frac{n-\lambda}{q}\right)}+C_{5} K_{1} \int_{(1-\epsilon) t}^{t}(t-\tau)^{-\frac{1}{2 \sigma}} \tau^{\frac{1-2 \sigma}{2 \sigma}}\left\|\left(\nabla^{m} u, \nabla^{m} d\right)\right\|_{q, \lambda} d \tau .
$$

Similarly, from a computation it follows that

$$
\left\|\nabla^{m} d(t)\right\|_{q, \lambda} \leq b_{\epsilon} t^{-\frac{m}{2 \sigma}-\frac{1}{2 \sigma}\left(2 \sigma-1-\frac{n-\lambda}{q}\right)}+C_{5} K_{1} \int_{(1-\epsilon) t}^{t}(t-\tau)^{-\frac{1}{2 \sigma}} \tau^{\frac{1-2 \sigma}{2 \sigma}}\left\|\left(\nabla^{m} u, \nabla^{m} d\right)\right\|_{q, \lambda} d \tau .
$$

Thus, we have

$$
\begin{aligned}
& \left\|\left(\nabla^{m} u(t), \nabla^{m} d(t)\right)\right\|_{q, \lambda} \\
& \quad \leq b_{\epsilon} t^{-\frac{m}{2 \sigma}-\frac{1}{2 \sigma}\left(2 \sigma-1-\frac{n-\lambda}{q}\right)}+C_{5} K_{1} \int_{(1-\epsilon) t}^{t}(t-\tau)^{-\frac{1}{2 \sigma}} \tau^{\frac{1-2 \sigma}{2 \sigma}}\left\|\left(\nabla^{m} u, \nabla^{m} d\right)\right\|_{q, \lambda} d \tau .
\end{aligned}
$$

Applying Lemma 2.5, we see that there exists $\epsilon_{m_{0}} \in(0,1)$, such that for any $0<\epsilon_{m} \leq \epsilon_{m_{0}}$, we have

$$
\left\|\left(\nabla^{m} u(t), \nabla^{m} d(t)\right)\right\|_{q, \lambda} \leq 2 b_{\epsilon_{m}} t^{-\frac{m}{2 \sigma}-\frac{1}{2 \sigma}\left(2 \sigma-1-\frac{n-\lambda}{q}\right)}
$$


where $I\left(\epsilon_{m_{0}}\right)=\min \left\{\frac{1}{2 C_{5} K_{1}}, I(1)\right\}$. Let $\epsilon_{m}=\frac{1}{m^{2 \sigma}}$, since $I(\epsilon)$ is nonincreasing with respect to $\epsilon$. We can choose $m_{0}>2$ sufficiently large such that $I\left(\frac{1}{m^{2 \sigma}}\right) \leq \frac{1}{2 C_{5} K_{1}}$ for all $m \geq m_{0}$. Hence, we obtain

$$
\left\|\left(\nabla^{m} u(t), \nabla^{m} d(t)\right)\right\|_{q, \lambda} \leq 2 b_{\frac{1}{m^{2 \sigma}}} t^{-\frac{m}{2 \sigma}-\frac{1}{2 \sigma}\left(2 \sigma-1-\frac{n-\lambda}{q}\right)} .
$$

By (3.17), we can choose $K_{1}$ and $K_{2}$ sufficiently large such that (3.2) holds for all $|\tilde{\beta}| \leq m_{0}$. Finally, it is enough to show that $2 b_{\frac{1}{m^{2 \sigma}}} \leq K_{1}\left(K_{2}|\tilde{\beta}|\right)^{2|\tilde{\beta}|-\delta}$ for any $m>m_{0} \geq 2$ with constants $K_{1}$ and $K_{2}$ sufficiently large.

Next, we compute $I\left(\frac{1}{m^{2 \sigma}}\right)$,

$$
\begin{aligned}
I\left(\frac{1}{m^{2 \sigma}}\right) & \leq \int_{1-\frac{1}{m^{2 \sigma}}}^{1}(1-\tau)^{-\frac{1}{2 \sigma}} \tau^{-\frac{m}{2 \sigma}-\frac{1}{2 \sigma}\left(4 \sigma-2-\frac{n-\lambda}{q}\right)} d \tau \\
& \leq \int_{1-\frac{1}{m^{2 \sigma}}}^{1}(1-\tau)^{-\frac{1}{2 \sigma}} d \tau\left(1-\frac{1}{m^{2 \sigma}}\right)^{-\frac{m}{2 \sigma}}\left(1-\frac{1}{m^{2 \sigma}}\right)^{-\frac{1}{2 \sigma}\left(4 \sigma-2-\frac{n-\lambda}{q}\right)} \\
& \leq \frac{2 \sigma}{2 \sigma-1} m_{0}^{1-2 \sigma} e^{\frac{m_{0}^{1-2 \sigma}}{2 \sigma}}\left(1-\frac{1}{m_{0}^{2 \sigma}}\right)^{-\frac{1}{2 \sigma}(4 \sigma-2)} \\
& \leq \frac{2 \sigma}{2 \sigma-1} 2^{1-2 \sigma} e^{\frac{2^{1-2 \sigma}}{2 \sigma}}\left(1-\frac{1}{2^{2 \sigma}}\right)^{-\frac{1}{2 \sigma}(4 \sigma-2)} \\
& \leq C(\sigma)
\end{aligned}
$$

Since $\delta \leq 2 m-\frac{m}{2 \sigma}\left(\frac{1}{2}<\delta \leq 1, m \geq 2, \frac{1}{2}<\sigma<2\right), r>n-\lambda, \frac{1}{2}+\frac{n-\lambda}{4 r}<\sigma<1+\frac{n-\lambda}{4 r}<2$, and $m^{\delta+2} \leq 8 \cdot 2^{m-\delta} \sqrt{m} \leq 8 \cdot 2^{2 m-\delta}$, we thus have

$$
\begin{aligned}
2 b_{\frac{1}{m^{2 \sigma}} \leq} \leq & \left\{C_{1} M_{1} C_{2}^{2 m-\delta} m^{2 m-\delta}+C_{3} M_{2}^{2} C_{4}^{\frac{m}{2 \sigma}} m^{\frac{m}{2 \sigma}}\left(\frac{1}{m^{2 \sigma}}\right)^{-\frac{m+1}{2 \sigma}-\frac{n-\lambda}{2 \sigma}\left(\frac{1}{r}-\frac{1}{q}\right)}\right. \\
& \left.+C_{6} C(\sigma) K_{1}^{2} K_{2}^{2 m-2 \delta} m^{2 m-\delta}\right\} \\
\leq & 2\left\{C_{1} M_{1} C_{2}^{2 m-\delta} m^{2 m-\delta}+C_{3} M_{2}^{2} C_{4}^{\frac{m}{2 \sigma}} m^{\frac{m}{2 \sigma}} m^{m+1+\frac{n-\lambda}{r}-\frac{n-\lambda}{q}}\right. \\
& \left.+C_{6} C(\sigma) K_{1}^{2} K_{2}^{2 m-2 \delta} m^{2 m-\delta}\right\} \\
\leq & 2\left\{C_{1} M_{1} C_{2}^{2 m-\delta} m^{2 m-\delta}+C_{3} M_{2}^{2} C_{4}^{2 m-\delta} m^{2 m-\delta} m^{\delta+2}+C_{6} C(\sigma) K_{1}^{2} K_{2}^{2 m-2 \delta} m^{2 m-\delta}\right\} \\
\leq & 2\left\{C_{1} M_{1} C_{2}^{2 m-\delta} m^{2 m-\delta}+C_{3} M_{2}^{2} C_{4}^{2 m-\delta} m^{2 m-\delta} 8 \cdot 2^{2 m-\delta}\right. \\
& \left.+C_{6} C(\sigma) K_{1}^{2} K_{2}^{2 m-2 \delta} m^{2 m-\delta}\right\} \\
\leq & 2\left\{C_{1} M_{1} C_{2}^{2 m-\delta}+C_{3} M_{2}^{2} C_{4}^{2 m-\delta} 2^{2 m-\delta}+C_{6} C(\sigma) K_{1}^{2} K_{2}^{2 m-2 \delta}\right\} m^{2 m-\delta} .
\end{aligned}
$$

We choose the constants $K_{1}:=4 C_{1} M_{1}+4 C_{3} M_{2}^{2}$. We take $K_{2}$ large enough, such that $K_{2} \geq$ $2 C_{4}+C_{2}$ and $C_{6} C(\sigma) K_{1} K_{2}^{-\delta}<\frac{1}{2}$. Then we obtain (3.2) immediately.

Proposition 3.2 Suppose that the assumptions of Theorem 1.1 are satisfied. Then the mild solution $(u, d)$ of (1.2) satisfies (3.1), and there exist constants $\tilde{K}_{1}, \tilde{K}_{2}>0$ such that

$$
\left\|\left(\partial_{x}^{\tilde{\beta}} u, \partial_{x}^{\tilde{\beta}} d\right)\right\|_{q, \lambda} \leq \tilde{K}_{1}\left(\tilde{K}_{2} m\right)^{2 m} t^{-\frac{m}{2 \sigma}-\frac{1}{2 \sigma}\left(2 \sigma-1-\frac{n-\lambda}{q}\right)}
$$

for all $r \leq q \leq \infty$, where $|\tilde{\beta}|=m$. 
Proof The proof is now standard, we refer the reader to [10-12].

Now Theorem 1.1 follows immediately from Proposition 3.1 and Proposition 3.2. We thus complete the proof of Theorem 1.1.

\section{Appendix}

In this Appendix, we will show the global existence of solution for system (1.1) mentioned in Theorem 1.1 or (I) of Remarks below Theorem 1.1. We note that, similarly to the Navier-Stokes equations ([3], Theorems 3 and 4, p.967), the proof of global existence can be obtained by making minor modifications to Theorems 3 and 4 on p.967 in [3]. Here, we give a brief argument of this proof for completeness and for convenience of the reader.

We say $(u, d) \in E^{\epsilon_{0}} \times E^{\epsilon_{0}}$ if $(u, d) \in E \times E$ and $\|(u, d)\|_{E^{\epsilon_{0}}}=\|(u, d)\|_{E}=\sup _{t>0} \|(u(t)$, $d(t))\left\|_{\dot{\mathrm{N}}_{r, \lambda, \infty}^{-s}}+\sup _{t>0} t^{\frac{\alpha}{2}}\right\|(u(t), d(t)) \|_{2 r, \lambda} \leq C \epsilon_{0}$. The definition of $E$ can be found in (I) of Remarks below Theorem 1.1.

Lemma A.1 Let $n \geq 2,1 \leq r<\infty, 0 \leq \lambda<n, r>n-\lambda, \frac{1}{2}+\frac{n-\lambda}{4 r}<\sigma<1+\frac{n-\lambda}{4 r}, s=2 \sigma-1-\frac{n-\lambda}{r}$, $\alpha=\frac{2 \sigma-1}{\sigma}-\frac{n-\lambda}{2 r \sigma}, \nabla \cdot u_{0}=0, \nabla \cdot d_{0}=0,\left(u_{0}, d_{0}\right) \in \dot{\mathbf{N}}_{r, \lambda, \infty}^{-s} \times \dot{\mathbf{N}}_{r, \lambda, \infty}^{-s}, q \in[r, \infty]$. There exists a constant $M_{1}>0$, such that $\left(u_{0}, d_{0}\right)$ satisfies (1.5), then we have $\left(\bar{u}_{0}, \bar{d}_{0}\right) \in E_{M_{1}} \times E_{M_{1}}$, where $\bar{u}_{0}=e^{-t \mathfrak{L}} u_{0}$ and $\bar{d}_{0}=e^{-t \mathfrak{L}} d_{0}$.

Proof From (2.3) of Lemma 2.2, we thus obtain

$$
\left\|\left(\bar{u}_{0}, \bar{d}_{0}\right)\right\|_{\dot{\mathbf{N}}_{r, \lambda, \infty}^{-s}} \lesssim\left\|\left(u_{0}, d_{0}\right)\right\|_{\dot{\mathbf{N}}_{r, \lambda, \infty}^{-s}} .
$$

Note that $\alpha \sigma+\frac{n-\lambda}{2 r}=s+\frac{n-\lambda}{r}$, it follows from Lemma 2.3 and a Sobolev-type embedding of Lemma 2.1 that

$$
\sup _{t>0} t^{\frac{\alpha}{2}}\left\|\left(\bar{u}_{0}, \bar{d}_{0}\right)\right\|_{2 r, \lambda} \cong\left\|\left(u_{0}, d_{0}\right)\right\|_{\dot{\mathrm{N}}_{r, \lambda, \lambda, \infty}^{-\alpha \sigma}} \lesssim\left\|\left(u_{0}, d_{0}\right)\right\|_{\dot{\mathrm{N}}_{r, \lambda, \infty}^{-s}}
$$

Hence, the proof of Lemma A.1 is now completed.

Define

$$
\left\{\begin{array}{l}
\Phi_{1}(u, d)=-\int_{0}^{t} e^{-(t-s) \mathfrak{L}} \mathbb{P} \nabla \cdot(u \otimes u-d \otimes d)(\cdot, s) d s \\
\Phi_{2}(u, d)=-\int_{0}^{t} e^{-(t-s) \mathfrak{L}} \mathbb{P} \nabla \cdot(u \otimes d-d \otimes u)(\cdot, s) d s .
\end{array}\right.
$$

Lemma A.2 Let $n \geq 2,1 \leq r<\infty, 0 \leq \lambda<n, r>n-\lambda, \frac{1}{2}+\frac{n-\lambda}{4 r}<\sigma<1+\frac{n-\lambda}{4 r}, s=2 \sigma-1-\frac{n-\lambda}{r}$, $\alpha=\frac{2 \sigma-1}{\sigma}-\frac{n-\lambda}{2 r \sigma}$. $\Phi_{1}$ and $\Phi_{2}$ were defined by (A.3), respectively. It holds true that

$$
\begin{aligned}
& \sup _{t>0}\left\|\left(\Phi_{1}(u, d), \Phi_{2}(u, d)\right)\right\|_{\dot{\Gamma}_{r, \lambda, \infty}^{-s}} \lesssim\|(u, d)\|_{E^{\prime}}^{2} \\
& \sup _{t>0} t^{\frac{\alpha}{2}}\left\|\left(\Phi_{1}(u, d), \Phi_{2}(u, d)\right)\right\|_{2 r, \lambda} \lesssim\|(u, d)\|_{E}^{2}
\end{aligned}
$$

for all $(u, d) \in E \times E$. 
Proof From Lemmas 2.1 and 2.2, it follows that

$$
\begin{aligned}
& \left\|\Phi_{1}(u, d)\right\|_{\dot{\mathbf{N}}_{r, \lambda, \infty}^{-s}} \lesssim \int_{0}^{t}(t-s)^{\frac{s-1}{2 \sigma}}\|\mathbb{P} \nabla \cdot(u \otimes u-d \otimes d)(\cdot, s)\|_{\dot{\mathbf{N}}_{r, \lambda, \infty}^{-1}} d s \\
& \lesssim \int_{0}^{t}(t-s)^{\frac{s-1}{2 \sigma}}\|(u \otimes u-d \otimes d)(\cdot, s)\|_{\dot{\mathbf{N}}_{r, \lambda, \infty}^{0}} d s \\
& \lesssim \int_{0}^{t}(t-s)^{\frac{s-1}{2 \sigma}}\|(u \otimes u-d \otimes d)(\cdot, s)\|_{r, \lambda} d s \\
& \lesssim \int_{0}^{t}(t-s)^{\frac{s-1}{2 \sigma}}\left(\|u(\cdot, s)\|_{2 r, \lambda}^{2}+\|d(\cdot, s)\|_{2 r, \lambda}^{2}\right) d s \\
& \lesssim \int_{0}^{t}(t-s)^{\frac{s-1}{2 \sigma}} s^{-\alpha} d s\left(\|u\|_{E}^{2}+\|d\|_{E}^{2}\right) \\
& \lesssim\|(u, d)\|_{E}^{2} .
\end{aligned}
$$

By Lemmas 2.1, 2.2 and 2.3, we obtain the estimate $t^{\frac{\alpha}{2}}\left\|\Phi_{1}(u, d)\right\|_{2 r, \lambda} \lesssim t^{\frac{\alpha}{2}}\left\|\Phi_{1}(u, d)\right\|_{\dot{\mathbf{N}}_{2 r, \lambda, 1}^{0}} \lesssim$ $t^{\frac{\alpha}{2}}\left\|\Phi_{1}(u, d)\right\|_{\dot{\mathbf{N}}_{r, \lambda, 1}^{2 \sigma-1-\alpha \sigma} \text {. We thus obtain }}$

$$
\begin{aligned}
t^{\frac{\alpha}{2}}\left\|\Phi_{1}(u, d)\right\|_{2 r, \lambda} & \lesssim t^{\frac{\alpha}{2}} \int_{0}^{t}(t-s)^{\frac{\alpha-2}{2}}\|\mathbb{P} \nabla \cdot(u \otimes u-d \otimes d)(\cdot, s)\|_{\dot{\mathbf{N}}_{r, \lambda, \infty}^{-1}} d s \\
& \lesssim t^{\frac{\alpha}{2}} \int_{0}^{t}(t-s)^{\frac{\alpha-2}{2}}\|\mathbb{P}(u \otimes u-d \otimes d)(\cdot, s)\|_{\dot{\mathbf{N}}_{r, \lambda, \infty}^{0}} d s \\
& \lesssim t^{\frac{\alpha}{2}} \int_{0}^{t}(t-s)^{\frac{\alpha-2}{2}}\left(\|u(\cdot, s)\|_{2 r, \lambda}^{2}+\|d(\cdot, s)\|_{2 r, \lambda}^{2}\right) d s \\
& \lesssim t^{\frac{\alpha}{2}} \int_{0}^{t}(t-s)^{\frac{\alpha-2}{2}} s^{-\alpha} d s\left(\|u\|_{E}^{2}+\|d\|_{E}^{2}\right) \\
& \lesssim\|(u, d)\|_{E}^{2}
\end{aligned}
$$

In the following, in a similar way to the derivation of (A.4) and (A.5), we have

$$
\begin{aligned}
& \left\|\Phi_{2}(u, d)\right\|_{\dot{\mathbf{N}}_{r, \lambda, \infty}^{-s}} \lesssim\|u\|_{E}\|d\|_{E} \lesssim\|(u, d)\|_{E^{\prime}}^{2} \\
& t^{\frac{\alpha}{2}}\left\|\nabla \Phi_{2}(u, d)\right\|_{2 r, \lambda} \lesssim\|u\|_{E}\|d\|_{E} \lesssim\|(u, d)\|_{E^{*}}^{2}
\end{aligned}
$$

Thus, we complete the proof of Lemma A.2.

Lemma A.3 Let $n \geq 2,1 \leq r<\infty, 0 \leq \lambda<n, r>n-\lambda, \frac{1}{2}+\frac{n-\lambda}{4 r}<\sigma<1+\frac{n-\lambda}{4 r}, s=2 \sigma-1-\frac{n-\lambda}{r}$, $\alpha=\frac{2 \sigma-1}{\sigma}-\frac{n-\lambda}{2 r \sigma}, \nabla \cdot u_{0}=0, \nabla \cdot d_{0}=0,\left(u_{0}, d_{0}\right) \in \dot{\mathbf{N}}_{r, \lambda, \infty}^{-s} \times \dot{\mathbf{N}}_{r, \lambda, \infty}^{-s}, q \in[r, \infty]$. Given a constant $M_{2}>0$ small enough, let $(\hat{u}, \hat{d}) \in E_{M_{2}} \times E_{M_{2}}$, and $\left(u_{0}, d_{0}\right)$ satisfy $(1.5)$, then $(u, d) \in E_{M_{2}} \times$ $E_{M_{2}}$, where

$$
\begin{aligned}
& u=e^{-t \mathfrak{L}} u_{0}-\int_{0}^{t} e^{-(t-s) \mathfrak{L}} \mathbb{P} \nabla \cdot(\hat{u} \otimes \hat{u}-\hat{d} \otimes \hat{d})(\cdot, s) d s, \\
& d=e^{-t \mathfrak{L}} d_{0}-\int_{0}^{t} e^{-(t-s) \mathfrak{L}} \mathbb{P} \nabla \cdot(\hat{u} \otimes \hat{d}-\hat{d} \otimes \hat{u})(\cdot, s) d s .
\end{aligned}
$$


Proof We will prove $(u, d) \in E_{M_{2}} \times E_{M_{2}}$. Due to Lemmas A.1 and A.2, one thus has

$$
\begin{aligned}
\|(u, d)\|_{E} \lesssim & \left\|\left(e^{-t \mathfrak{L}} u_{0}, e^{-t \mathfrak{L}} d_{0}\right)\right\|_{E}+\sup _{t>0}\left\|\left(\Phi_{1}(\hat{u}, \hat{d}), \Phi_{2}(\hat{u}, \hat{d})\right)\right\|_{\dot{\mathrm{N}}_{r, \lambda, \infty}^{-\beta}} \\
& +\sup _{t>0} t^{\frac{\alpha}{2}}\left\|\left(\Phi_{1}(\hat{u}, \hat{d}), \Phi_{2}(\hat{u}, \hat{d})\right)\right\|_{2 r, \lambda} \\
\leq & C_{1} M_{1}+C_{2} M_{2}^{2} \\
\leq & C M_{2}
\end{aligned}
$$

provided $0<M_{1} \leq M_{2}$ is chosen to be sufficiently small, where we have used the estimate

$$
C_{1} M_{1}+C_{2} M_{2}^{2} \leq M_{2}\left(C_{1}+C_{1} M_{2}\right) \leq M_{2}\left(C_{1}+C_{2}\right)=C M_{2}
$$

in the last step. Therefore, we obtain $(u, d) \in E_{M_{2}} \times E_{M_{2}}$.

Hence, the proof of Lemma A.3 is finished.

Lemma A.4 For all $M_{2}>0$ small enough, let $(u, d) \in E_{M_{2}} \times E_{M_{2}}$ and $(\tilde{u}, \tilde{d}) \in E_{M_{2}} \times E_{M_{2}}$ with the same initial data $\left(u_{0}, d_{0}\right)$, then $\Phi=\left[\Phi_{1}, \Phi_{2}\right]$ defined in (A.3) is a contractive map.

Proof Let $\mathfrak{u}=u-\tilde{u}$ and $\mathfrak{d}=d-\tilde{d}$, repeating the proof as Lemma A.2, it holds true that

$$
\begin{aligned}
\left\|\Phi_{1}(u, d)-\Phi_{1}(\tilde{u}, \tilde{d})\right\|_{E_{M_{2}}} & \lesssim\left(\|\tilde{u}\|_{E_{M_{2}}}+\|u\|_{E_{M_{2}}}\right)\|\mathfrak{u}\|_{E_{M_{2}}}+\left(\|\tilde{d}\|_{E}+\|d\|_{E_{M_{2}}}\right)\|\mathfrak{d}\|_{E_{M_{2}}} \\
& \leq C M_{2}\left(\|\mathfrak{u}\|_{E_{M_{2}}}+\|\mathfrak{d}\|_{E_{M_{2}}}\right) .
\end{aligned}
$$

Meanwhile, similar to the proof of Lemma A.2, we have

$$
\begin{aligned}
\left\|\Phi_{2}(u, d)-\Phi_{2}(\tilde{u}, \tilde{d})\right\|_{E_{M_{2}}} & \lesssim\left(\|\tilde{u}\|_{E_{M_{2}}}+\|u\|_{E_{M_{2}}}\right)\|\mathfrak{d}\|_{E_{M_{2}}}+\left(\|\tilde{d}\|_{E_{M_{2}}}+\|d\|_{E_{M_{2}}}\right)\|\mathfrak{u}\|_{E_{M_{2}}} \\
& \leq C M_{2}\left(\|\mathfrak{u}\|_{E_{M_{2}}}+\|\mathfrak{d}\|_{E_{M_{2}}}\right) .
\end{aligned}
$$

Taking $M_{2}>0$ small enough, there exists $0<\theta<\frac{1}{2}$, such that

$$
\left\|\Phi_{1}(u, d)-\Phi_{1}(\tilde{u}, \tilde{d})\right\|_{E_{M_{2}}}+\left\|\Phi_{2}(u, d)-\Phi_{2}(\tilde{u}, \tilde{d})\right\|_{E_{M_{2}}} \leq \theta\left(\|d-\tilde{d}\|_{E_{M_{2}}}+\|u-\tilde{u}\|_{E_{M_{2}}}\right) .
$$

Therefore, $\Phi=\left[\Phi_{1}, \Phi_{2}\right]$ is a contractive map and we complete the proof of Lemma A.4.

Applying Banach's fixed pointed theorem, we finish the proof of global existence, it following directly from Lemmas A.1, A.2, A.3, and A.4.

\section{Competing interests}

The author declares to have no competing interests.

\section{Acknowledgements}

The author is highly grateful for the referees' careful reading of and comments on this paper. The author would like to thank Professor Lixin Yan for helpful discussions.

Received: 17 March 2015 Accepted: 14 August 2015 Published online: 04 September 2015 


\section{References}

1. Wu, J: Generalized MHD equations. J. Differ. Equ. 195, 284-312 (2003)

2. Kato, T: Strong solutions of the Navier-Stokes equation in Morrey spaces. Bol. Soc. Bras. Mat. 22(2), 127-155 (1992)

3. Kozono, H, Yamazaki, M: Semilinear heat equations and the Navier-Stokes equation with distributions in new function spaces as initial data. Commun. Partial Differ. Equ. 19, 959-1014 (1994)

4. Hajer, B, Chemin, YJ, Danchin, R: Fourier Analysis and Nonlinear Partial Differential Equations. Springer, Berlin (2011)

5. Foias, C, Temam, R: Gevrey class regularity for the solutions of the Navier-Stokes equations. J. Funct. Anal. 87, 359-369 (1989)

6. Grujič, Z, Kukavica, I: Space analyticity for the Navier-Stokes and related equations with initial data in $L^{p}$. J. Funct. Anal. $152,247-466(1998)$

7. Lemarié-Rieusset, PG: On the analyticity of mild solutions for the Navier-Stokes equations. C. R. Acad. Sci. Paris, Ser. I 330, 183-186 (2000)

8. Kahane, C: On the spatial analyticity of solutions of the Navier-Stokes equations. Arch. Ration. Mech. Anal. 33, 386-405 (1969)

9. Komatsu, G: Global analyticity up to the boundary of solutions of the Navier-Stokes equation. Commun. Pure Appl. Math. 33(4), 545-566 (1980)

10. Giga, Y, Sawada, O: On regularizing-decay rate estimates for solutions to the Navier-Stokes initial value problem. In: Nonlinear Analysis and Applications: To V. Lakshmikantham on His 80th Birthday, vols. 1,2, pp. 549-562 (2003)

11. Sawada, O: On analyticity rate estimates of the solutions to the Navier-Stokes equations in Bessel potential spaces. J. Math. Anal. Appl. 312, 1-13 (2005)

12. Miura, $\mathrm{H}$, Sawada, $\mathrm{O}$ : On the regularizing rate estimates of Koch-Tataru's solution to the Navier-Stokes equations. Asymptot. Anal. 49, 1-15 (2006)

13. Bae, H, Biswas, A, Tadmor, E: Analyticity and decay estimates of the Navier-Stokes equations in critical Besov spaces. Arch. Ration. Mech. Anal. 205(3), 963-991 (2012)

14. Huang, C, Wang, B: Analyticity for the (generalized) Navier-Stokes equations with rough initial data. arXiv:1310.2141

15. Dong, $\mathrm{H}, \mathrm{Li}$, D: Optimal local smoothing and analyticity rate estimates for the generalized Navier-Stokes equations. Commun. Math. Sci. 7, 67-80 (2009)

16. Liu, Q, Cui, SB: Regularizing rate estimates for mild solutions of the incompressible magnetohydrodynamic system. Commun. Pure Appl. Anal. 11(5), 1643-1660 (2012)

17. Liu, Q, Zhao, JH, Cui, SB: Existence and regularizing rate estimates of solutions to a generalized magneto-hydrodynamic system in pseudomeasure spaces. Ann. Mat. Pura Appl. 191, 293-309 (2012)

18. Yamamoto, M: Spatial analyticity of solutions to the drift-diffusion equation with generalized dissipation. Arch. Math. 97, 261-270 (2011)

19. Mazzucato, A: Besov-Morrey spaces function space theory and applications to non-linear PDE. Trans. Am. Math. Soc. 355(4), 1297-1364 (2003)

20. Lucas, F, Juliana, C: Existence of solutions for the 3D-micropolar fluid system with initial data in Besov-Morrey spaces. Z. Angew. Math. Phys. 64, 1699-1710 (2013)

21. Yuan, W, Haroske, D, Moura, D, Skrzypczak, L, Yang, D: Limiting embeddings in smoothness Morrey spaces, continuity envelopes and applications. J. Approx. Theory (2015). doi:10.1016/j.jat.2014.12.006

22. Tang, L: A remark on the well-posedness of the Euler equation in the Besov-Morrey space. http://162.105.204.96/var/preprint/572.pdf

23. Lucas, F, Villamizar, J: Fractional Navier-Stokes equations and a Hölder-type inequality in a sum of singular spaces. Nonlinear Anal. 74, 5618-5630 (2011)

24. Cannone, M, Karch, G: Smooth or singular solutions to the Navier-Stokes system. J. Differ. Equ. 197(2), 247-274 (2004)

25. Koch, H, Tataru, D: Well-posedness for the Navier-Stokes equations. Adv. Math. 157, 22-35 (2001)

26. Almeida, M, Ferreira, L: On the Navier-Stokes equations in the half-space with initial and boundary rough data in Morrey spaces. J. Differ. Equ. 254, 1548-1570 (2013)

27. Taylor, M: Analysis on Morrey spaces and applications to Navier-Stokes and other evolution equations. Commun. Partial Differ. Equ. 17, 1407-1456 (1992)

28. Miao, C, Yuan, B, Zhang, B: Well-posedness of the Cauchy problem for the fractional power dissipative equations. Nonlinear Anal. 68, 461-484 (2008)

29. Shibata, Y, Shimizu, S: A decay property of the Fourier transform and its application to the Stokes problem. J. Math. Fluid Mech. 3(3), 213-230 (2001)

30. Miao, C, Wu, J, Zhang, Z: Littlewood-Paley Theory and Applications to Fluid Dynamics Equations. Monogr. Modern Pure Math., vol. 142. Science Press, Beijing (2012) 